\title{
Profit-maximizing operation and valuation of hydroelectric plant: a new solution to the Koopmans problem*
}

\author{
Anthony Horsley and Andrew J. Wrobel \\ Department of Economics, London School of Economics \\ Houghton Street, London WC2A 2AE, United Kingdom. \\ E-mail: LSEecon123@mac.com
}

21 February 2005

CDAM Research Report LSE-CDAM-2005-03

\begin{abstract}
Koopmans's approach to hydroelectric storage is reformulated for competitive profit maximization, with electricity priced by time of use. Duality methods of linear programming are applied to the problems of operation and rental valuation of a hydro plant and its river. Both problems are approached by using time-dependent shadow-pricing of water, and if the given market price for electricity $(p)$ is a continuous function of time, then the shadow price function for water $(\psi)$ is shown to be unique. The two prices $(\psi$ and $p)$ determine not only the optimal water storage policy but also the marginal values of the plant's capacities, defined as derivatives of the operating profit. In particular, the unit reservoir rent equals the total positive variation of the water value $(\psi)$ over the cycle. Profit-imputed values of the river flow and of the hydro capacities (reservoir and turbine) are therefore definiteunlike Koopmans's cost-imputed values, derived from fuel savings in a mixed hydro-thermal system. The marginal capacity values can be used to determine the optimum investment. The model applies also to other natural flows, e.g., water supply and geothermal or tidal energy.
\end{abstract}

Keywords: Storage hydro, peak-load pricing, marginal values, linear programming, subdifferential calculus.

2000 AMS Mathematics Subject Classification: Primary 90B05. Secondary 90C08, 90C31.

1991 Journal of Economic Literature Classification: C61, D24, L94, L95.

\footnotetext{
${ }^{*}$ Financial support of the ESRC is gratefully acknowledged (grant R000232822). We also thank the referees and the editor for their comments.
} 


\section{Introduction}

In view of the economic significance of hydroelectric generation to many countries, the scale of the investment it can entail, and the planning and operational difficulties it presents, it is unsurprising that it is the subject of so much study by engineers, operations researchers and economists. A common economic understanding should inform this work and, indeed, in his 1957 article [20], and again in his 1975 Nobel Lecture [21, pp. 262-263], Koopmans pointed to the efficiency rents of the fixed inputs (river flow, reservoir and turbine) as the elements that can underpin the various approaches. In fact, the models of different researchers have remained largely separate, ${ }^{1}$ and this is because of the technical obstacles faced by economists in taking their part of the project forward - for although Koopmans's work is much cited, it has never been used in practice or, until now, followed up in theory.

We recast Koopmans's operation problem as one of competitive profit maximization, which is the relevant setting for modern decentralized electricity supply industries. Several advances in mathematical economics instruct our solution, and Koopmans's continuous-time formulation can now be handled as part of a general equilibrium problem in an infinite-dimensional commodity space. The framework we use is the adaptation of Bewley's equilibrium model [13] that we have developed to investigate Boiteux's conjectures on the peak-load pricing of electricity [5, 3.4 and 3.3.3]. Koopmans's scheme, like Boiteux's, is marginalist, and both encounter the problem of nondifferentiability of joint cost functions. We employ subdifferentials to describe multi-valued derivatives and have worked out generalizations of the smooth-calculus results that economists commonly use, including a subdifferential version of the Wong-Viner Envelope Theorem on the equality of short-run and long-run marginal costs $[16] .{ }^{2}$ The short-run approach to long-run general equilibrium that we have devised in [16] is the wider conceptual setting for this paper. This is because a key element of the short-run approach is the profit-based valuation of capital inputs.

Koopmans undertakes the task of minimizing the operating cost of an entire electrical system by constructing a water storage plan for the hydro-plant operation that minimizes the fuel cost incurred by the thermal generating plant in producing a given output of electricity. From this operating solution he imputes time-of-use (TOU) values both to electricity $(p)$ and to water $(\psi)$, and thence the two hydro capacities, viz., the reservoir and the generator. These shadow prices enable him to verify that his water storage plan is optimal. His objectives are of particular interest to a centralized utility (with a predominantly thermal system) that seeks efficient utilization of its plant and needs to calculate the marginal costs of electricity in its system. However, he adds greatly to his difficulties by setting out to infer all the values associated with the hydro plant (the shadow prices of electricity, water and the hydro capacities) in one fell swoop. As we show in [16], it is much simpler to split the complex problem of simultaneous

\footnotetext{
${ }^{1}$ In [12, Sections 5 and 6], we discuss Koopmans's analysis in more detail, and we review other work on cost minimisation for a hydro-thermal system, including [7, Chapters 5 and 6], [19] and [22]. See also the overview in [1, pp. 277-282]. A more recent operational study of hydro in [3], [9] and [23] is set up as a profit maximisation problem for a single hydro plant, but it concentrates entirely on operation and does not address the economic questions of valuation and investment (it makes no use of the dual solution $\psi$, except as a tool for deriving the primal operating solution, and does not point to the interpretation of $\psi$ as the marginal value of water).

${ }^{2}$ See also [10] for subdifferentiation of symmetric functions, such as the thermal generation cost (as a function of the output bundle).
} 
valuation of both outputs and fixed inputs into subproblems, one of which entails short-run profit calculations - even though profit maximization may not be the explicit operational objective. ${ }^{3}$ Furthermore, Koopmans's method has little or no place in today's largely deregulated and decentralized supply industry in which each plant aims, in the face of a time-dependent market price for electricity $p(t)$, to maximize its own profit (as opposed to participating in the collective cost-minimizing operation of a system of plants).

Having profit maximization as the optimizing principle not only allows us to address the problems of a decentralized supply industry (as well as those of a centralized utility), but also it facilitates a full and simple solution. In particular: (i) profit-maximizing hydro operation and the dual problem of valuation can be handled (in the case of a constant hydrostatic head) by means of linear programmes (LPs), rather than the convex programmes (CPs) needed for Koopmans's cost minimization; (ii) one can depict simply the solutions to the operation and valuation LPs, which is not possible with Koopmans's operation CP or its dual; ${ }^{4}$ and (iii) profitimputed values of the hydro capacities and the river flow (i.e., their marginal contributions to the operating profit) turn out to be fully determinate - unlike Koopmans's cost-imputed values of the hydro inputs (i.e., the marginal savings on the thermal operating cost).

In our setup, the TOU electricity value $p(t)$ is a given market price; it is not an imputed shadow price as in Koopmans's analysis. Given $p$, we impute an optimal TOU value, $\psi(t)$, to water (or, more precisely, to the water's potential energy). This essentially solves the operation problem (Section 8): the hydro plant is operated just like a thermal plant with a time-varying "fuel" price $\psi(t)$. It makes sense, then, to value a hydro plant's capacities by their marginal contributions to the maximum operating profit, a.k.a. short-run profit $\Pi_{\mathrm{SR}}^{\mathrm{H}}$. These rents can be expressed in terms of the electricity and water values $p$ and $\psi$ (Theorem 9). For a hydro plant with a constant head, the shadow price for water $(\psi)$ can be determined from a linear programme (21)-(27) that is dual to the LP of profit-maximizing operation (13)-(17)..$^{5}$ By using the dual constraints to eliminate the dual variables other than $\psi$, the dual is reformulated as a convex but unconstrained programme of shadow pricing the water (33)-(35). This leads to a simple characterization of the solution: the optimal $\psi$ is obtained from $p$ by "shaving off" the local peaks of $p$ and "filling in" its troughs, and the optimal output $(y)$ follows from this (Section 3, Figures 1a and 1b).

\footnotetext{
${ }^{3}$ In terms of the sub- or super-differential, $\partial$ or $\widehat{\partial}$ - a generalised, multi-valued derivative of a convex or concave function - the split calculation uses the rule: $(p,-r) \in \partial_{y, k} C_{\mathrm{SR}}(y, k)$ if and only if both $p \in \partial_{y} C_{\mathrm{SR}}(y, k)$ and $r \in \widehat{\partial}_{k} \Pi_{\mathrm{SR}}(p, k)$, where $C_{\mathrm{SR}}$ is the operating a.k.a. short-run cost as a function of the output bundle $y$ and fixed-input bundle $k$, and $\Pi_{\mathrm{SR}}$ is the operating profit as a function of $k$ and the output price system $p$. If the joint marginal values are nonunique (i.e., $\partial_{y, k} C_{\mathrm{SR}}$ is multi-valued because $C_{\mathrm{SR}}$ is nondifferentiable) then, for a $p \in \partial_{y} C_{\mathrm{SR}}(y, k)$, the set $\widehat{\partial}_{k} \Pi_{\mathrm{SR}}(p, k)$ is generally a proper subset of $-\partial_{k} C_{\mathrm{SR}}(y, k)$, and it may even be a singleton (in which case the ordinary gradient vector $\nabla_{k} \Pi_{\mathrm{SR}}$ exists): indeed, this is so in Theorem 9 . That is why $\partial_{y, k} C_{\mathrm{SR}}$ does not factorise into the Cartesian product of $\partial_{y} C_{\mathrm{SR}}$ and $\partial_{k} C_{\mathrm{SR}}$, and why its calculation in terms of partial subdifferentials requires the function $\Pi_{\mathrm{SR}}$ (which is, by definition, a partial convex conjugate of $C_{\mathrm{SR}}$ ). Like all results on marginal values of optimisation programmes, the splitting of $\partial_{y, k} C_{\mathrm{SR}}$ can be reformulated in terms of programme solutions (in particular, any $r$ from $\widehat{\partial}_{k} \Pi_{\mathrm{SR}}$ can be obtained from the fixed-input valuation programme that is dual to the profit-maximising operation programme). This is also spelt out in [16].

${ }^{4}$ Since Koopmans does not do so, we state the dual CP in our review of his work in [12].

${ }^{5}$ The dual is the problem of minimising the plant's value subject to the constraints that decompose the given price $p(t)$ into the sum of values of the plant's capital services $(\kappa)$ and the Lagrange multipliers $(\nu)$ for the nonnegativity constraints on water stock and electricity output (plus a constant, $\lambda$ ).
} 
This last insight also makes it easier to identify a critical case of the dual solution: the imputed TOU value of water (the function $\psi$ ) is unique if the given TOU electricity price $(p)$ is a continuous function of time (Lemma 8). ${ }^{6}$ It then follows that the capital inputs (reservoir and turbine) also have definite marginal values $\left(\partial \Pi_{\mathrm{SR}}^{\mathrm{H}} / \partial k_{\mathrm{St}}\right.$ and $\left.\partial \Pi_{\mathrm{SR}}^{\mathrm{H}} / \partial k_{\mathrm{Tu}}\right)$. This is not so in Koopmans's cost-minimum framework because he has to value both flows (electricity and water), and the shadow-price pair $(p$ and $\psi$ ) is typically indeterminate (although, as our result shows, for each continuous $p$ there is just one $\psi$ ). With competitive profit maximization, the output price $(p)$ is unique simply because it is treated as given, and although a fixed-input's shadow price $(\psi)$ might still be indeterminate, this possibility can be excluded by a problemspecific argument (which in our case consists in examining the structure of Lagrange multipliers for the capacity constraints). This is a major advantage of the profit approach.

The imputed values of the hydro capacities and the river flow are useful in making investment decisions, whether to expand an existing plant or to develop a new hydro site. This is an end Koopmans envisaged for his cost-imputed values, but their nonuniqueness causes complications because, for example, it means that the incremental value of investment becomes nonadditive (superadditive) when two or more hydro inputs are being varied. Such calculations are made much simpler by using the profit-imputed values: being unique, they can be simply equated to the corresponding marginal costs of investment to determine the optimal capacities. Also, the dual LP (or the equivalent unconstrained CP) gives a simple and direct way of imputing the values. By contrast, Koopmans's values are derived from a tortuous operating solution. They do serve his immediate purpose - to verify the cost-optimality of his storage plan-but the nonuniqueness of his values is an obstacle to their use in practical investment planning.

Time-continuity of the electricity price $(p)$ is not only a natural assumption to make; it is also one that we verify for competitive equilibrium in the commodity space of bounded functions of time [15], where the price function is proved to be continuous for a class of problems that includes peak-load pricing with storage. ${ }^{7}$

Section 2 describes the hydro technology. Formal analysis (with proofs in the Appendix) is preceded by heuristics, in Section 3. In Sections 4 and 5, the short-run profit maximum problem and its dual are set up as linear programmes (LPs) which are doubly infinite: with continuous-time dating of commodities, the primal (13)-(17) contains continua of output and input variables (electricity $y$ and river flow $e$ ) as well as continua of dated capacity constraints (on the electricity flow $y$ and on the water stock $s$ ). The primal and the dual are shown to be soluble, and their (optimal) solutions are described in Section 6. In Section 7, the dual LP is reformulated as an unconstrained CP of shadow-pricing the water. In Sections 8 and 9, the optimal water price $(\hat{\psi})$ is shown to be unique if the given electricity price $p$ is continuous over time; and formulae are given, in terms of $p$ and $\hat{\psi}$, for the optimal output $(\hat{y})$ and for the profit derivatives that represent the marginal values of the reservoir and the turbine $\left(\partial \Pi_{\mathrm{SR}}^{\mathrm{H}} / \partial k_{\mathrm{St}}\right.$ and $\left.\partial \Pi_{\mathrm{SR}}^{\mathrm{H}} / \partial k_{\mathrm{Tu}}\right)$. Their use in investment calculations is also sketched. Finally, Section 10 indicates the changes needed when the policy of pure "coasting" $(y=e)$ is infeasible (i.e., when $e \not \leq k_{\mathrm{Tu}}$ ); the hydro plant's operation may then necessitate spillage.

\footnotetext{
${ }^{6}$ Continuity over time is the only assumption on $p$ that can, by itself, guarantee uniqueness of the imputed values of water and the hydro capacities (see [11] for a counterexample when $p$ is a discontinuous step function and so time is effectively a discrete variable).

${ }^{7}$ The application to pumped storage is fully worked out in [15]; the case of hydroelectric storage is similar.
} 
In [17], we extend the analysis, and especially the valuation method, to the case of a variable head. This requires the use of a controlled differential equation, and the optimization problem becomes nonlinear (although it remains convex). Another reason for presenting that case separately is that the "hydro" technology has other interpretations, in which there is no equivalent of head variability. For example, the model herein is applicable to water supply (when priced by TOU), as well as to other natural energy flows (e.g., geothermal or tidal).

\section{Hydro technology}

Hydro generation produces electricity, a nonstorable good with a cyclical demand and price, from a storable input of water. ${ }^{8}$ We assume that a water stock, up to the reservoir's capacity, $k_{\mathrm{St}}$, can be held at no running cost (or loss of stock). Water is stored at a height, called the head, which determines its potential energy. This is converted first to kinetic energy in penstocks (the conduits carrying water down from the reservoir to turbines), and then to electrical energy by a turbine-driven generator (or "turbine" for brevity). In [17], we deal with the variable-head case, but a constant head is assumed here (as a good approximation for high-head plants). Therefore the energy stock $s(t)$ is always in a constant proportion to the stored water volume, and it can be referred to as "water". Similarly, the rate of river inflow, $e(t)$, can be measured in terms of power (instead of volume per unit time). ${ }^{9}$

The turbine-generator's technical efficiency is also taken to be constant. ${ }^{10}$ The water stock can therefore be measured as the output it actually yields on conversion (i.e., in kWh of electrical energy). The turbine capacity, $k_{\mathrm{Tu}}$, is its maximum output rate (in $\mathrm{kW}$ of electrical power), i.e., in unit time a unit turbine can convert a unit of stock into a unit of output.

The river inflow $e$ is taken to be known with certainty. It varies periodically over time, and a common cycle for the water inflow and the electricity price is represented by an interval $[0, T]$ of the real line $\mathbb{R}$. The cycle is generally a year because of seasonal variation, i.e., $T=8760$ hours. The inflow function $e$ is usually continuous, but it suffices to assume that $e$ is essentially bounded.

Definition A real-valued measurable function $e:[0, T] \rightarrow \mathbb{R}$ is essentially bounded, with respect to the Lebesgue measure (meas), if $e$ is bounded on $[0, T] \backslash N$ for some set $N$ with meas $N=0$. Functions which are equal almost everywhere (a.e.) -i.e., differ only on a set of measure zero - are identified with each other. The vector space of all the equivalence classes of essentially bounded functions is denoted by $L^{\infty}[0, T]$. It is normed by the

\footnotetext{
${ }^{8}$ The model applies also to electricity generation from geothermal energy, and it can be adapted to tidal energy (although this requires changes because a tidal plant cannot generate at all times in the ebb-flow cycle). The model applies also to the supply of other goods, such as water (when priced by TOU): in that case, the inflow $e(t)$ is the rainfall collected in reservoirs, and its conversion to the consumable good consists in water purification and pumping to users.

${ }^{9}$ For example, with a penstock efficiency of $98 \%$ and a turbine-generator's efficiency of $85 \%$, a cubic metre of water at a height of $100 \mathrm{~m}$ yields $10^{3} \times 9.81 \times 10^{2} \times 0.98 \times 0.85 /\left(3600 \times 10^{3}\right) \approx 0.227 \mathrm{kWh}$ of electric energy, and a river inflow of $1000 \mathrm{~m}^{3} / \mathrm{s}$ is equivalent to $10^{3} \times 9.81 \times 10^{2} \times 0.98 \times 0.85 \approx 817 \mathrm{MW}$ (the factor 9.81 is the Earth's gravity in $\left.\mathrm{m} / \mathrm{s}^{2}\right)$.

${ }^{10}$ In reality the equipment is not perfectly divisible, and a turbine's efficiency varies with the load (typically between $85 \%$ and $95 \%$ ). A generator's efficiency is a constant of ca. $95 \%$.
} 


\begin{tabular}{|c|c|c|c|}
\hline $\begin{array}{l}\text { Role in prog- } \\
\text { ramme pair }\end{array}$ & Variable & Notation & Unit \\
\hline $\begin{array}{l}\text { Price data } \\
\text { (dual } \\
\text { parameters) }\end{array}$ & $\begin{array}{l}\text { electricity price } \\
\text { at time } t\end{array}$ & $p(t)$ & $\$ / \mathrm{kWh}$ \\
\hline $\begin{array}{l}\text { Quantity } \\
\text { data } \\
\text { (primal } \\
\text { parameters) }\end{array}$ & $\begin{array}{c}\text { reservoir capacity } \\
\text { water-stock floor } \\
\text { turbine capacity } \\
\text { electr. output floor } \\
\text { river inflow rate at } t \\
\text { top-up of stock } \\
\end{array}$ & $\begin{array}{c}k_{\mathrm{St}}(t)=\text { const. } \\
n_{\mathrm{St}}(t)=0 \\
k_{\mathrm{Tu}}(t)=\text { const. } \\
n_{\mathrm{Tu}}(t)=0 \\
e(t) \\
\zeta=0\end{array}$ & $\begin{array}{c}\$ / \mathrm{kWh} \\
\mathrm{kWh} \\
\mathrm{kW} \\
\mathrm{kW} \\
\mathrm{kW} \\
\mathrm{kWh} \\
\end{array}$ \\
\hline $\begin{array}{l}\text { Quantity } \\
\text { decisions } \\
\text { (primal } \\
\text { variables) }\end{array}$ & $\begin{array}{l}\text { hydroelectric output } \\
\text { (water discharge } \\
\text { rate) at time } t \\
\text { water stock } \\
\text { at time } 0 \text { or } T\end{array}$ & $y(t)$ & $\mathrm{kWh}$ \\
\hline $\begin{array}{l}\text { Derived } \\
\text { quantities }\end{array}$ & $\begin{array}{l}\text { water stock at time } t \\
\text { rate of outflow from } \\
\text { reservoir at time } t\end{array}$ & $\begin{array}{c}s(t):=s_{0}-\int_{0}^{t} y(\tau) \mathrm{d} \tau \\
f(t):=y(t)-e(t)\end{array}$ & $\mathrm{kWh}$ \\
\hline $\begin{array}{l}\text { Shadow } \\
\text { prices } \\
\text { (dual } \\
\text { decision } \\
\text { variables, } \\
\text { paired } \\
\text { to primal } \\
\text { parameters) }\end{array}$ & $\begin{array}{l}\text { unit reservoir value } \\
\text { on interval length } \mathrm{d} t \\
\text { value of stock floor } \\
\text { (nonnegativity) } \\
\text { unit turbine value } \\
\text { at time } t \\
\text { value of output floor } \\
\text { (nonnegativity) } \\
\text { water value at time } t \\
\text { water value at } 0 \text { or } T\end{array}$ & $\begin{array}{c}\kappa_{\mathrm{St}}(\mathrm{d} t) \\
\nu_{\mathrm{St}}(\mathrm{d} t) \\
\kappa_{\mathrm{Tu}}(t) \\
\nu_{\mathrm{Tu}}(t) \\
\psi(t) \\
\lambda\end{array}$ & $\begin{array}{l}\$ / \mathrm{kWh} \\
\$ / \mathrm{kWh} \\
\$ / \mathrm{kWh} \\
\$ / \mathrm{kWh} \\
\$ / \mathrm{kWh} \\
\$ / \mathrm{kWh}\end{array}$ \\
\hline $\begin{array}{l}\text { Derived } \\
\text { valuations }\end{array}$ & $\begin{array}{l}\text { total reservoir rent } \\
\text { for whole cycle }[0, T] \\
\text { total turbine rent }\end{array}$ & $\begin{array}{c}\kappa_{\mathrm{St}}[0, T]=\int_{0}^{T} \kappa_{\mathrm{St}}(\mathrm{d} t) \\
\int_{0}^{T} \kappa_{\mathrm{Tu}}(t) \mathrm{d} t\end{array}$ & $\$$ \\
\hline
\end{tabular}

Table 1: Notation. Some functions of time $\left(k_{\text {St }}\right.$, etc. $)$ are equated to constants. This indicates that they are constants in the original, unperturbed programme, but are perturbed with timevarying increments $\left(\Delta k_{\mathrm{St}}(t)\right.$, etc.) to interpret the time-dependent dual variables $\left(\Delta \kappa_{\mathrm{St}}\right.$, etc.). The duality scheme (Section 5) similarly uses a nonzero increment $\Delta \zeta$ to $\zeta=0$ (paired with the dual variable $\lambda)$. NB: $\psi(t)=\lambda+\left(\kappa_{\mathrm{St}}-\nu_{\mathrm{St}}\right)[0, t)$ by a constraint of the dual (valuation) LP. Also, Section 10 uses an extra primal variable $\sigma$ to denote spillage. 
supremum norm

$$
\|e\|_{\infty}=\operatorname{EssSup}|e|=\operatorname{ess} \sup _{t \in[0, T]}|e(t)|:=\inf _{N: \text { meas } N=0} \sup _{t \in[0, T] \backslash N}|e(t)| .
$$

For the use of $L^{\infty}$ as a commodity space in an equilibrium model of peak-load pricing, see [13] and [15]. For some other uses of $L^{\infty}$, with a general underlying measure (instead of meas), see [4].

The hydro plant's output rate is also a periodic function, $y_{\mathrm{H}}$ (abbreviated to $y$ ). A storage policy generally consists of an output $y(t) \geq 0$ and a spillage $\sigma(t) \geq 0$. However, except in Section 10, spillage is excluded by the assumption that $k_{\mathrm{Tu}} \geq e$. This makes it feasible for the plant to "coast", i.e., to generate at a rate $y(t)$ equal to the inflow rate $e(t)$. It also means that all the incentive to use the reservoir comes from a time-dependent output price: if $p$ were a constant, the plant might as well coast all the time.

The net outflow from the reservoir is the signed function

$$
f=y-e+\sigma
$$

and the stock, $s(t)$ at time $t$, is an absolutely continuous function on $[0, T]$ that satisfies the evolution equation $\dot{s}:=\mathrm{d} s / \mathrm{d} t=-f$. This can be restated as

$$
s(t)-s(0)=-\int_{0}^{t} f(\tau) \mathrm{d} \tau:=\int_{0}^{t}(-y+e-\sigma)(\tau) \mathrm{d} \tau .
$$

So $s$ is actually a Lipschitz function, since $k_{\mathrm{Tu}} \geq y \geq 0$ a.e., and since both $e$ and $\sigma$ are also bounded (by assumption).

The space of all continuous functions $\mathcal{C}[0, T]$, which contains the Lipschitz functions, is normed by the maximum norm

$$
\|s\|_{\infty}=\operatorname{Max}|s|=\max _{t \in[0, T]}|s(t)| .
$$

Its norm-dual $\mathcal{C}^{*}$, which serves as the price space for the services of storage capacity, is identified as the space of all (signed, finite) Borel measures $\mathcal{M}[0, T]$ by means of the bilinear form

$$
\langle\mu, s\rangle:=\int_{[0, T]} s(t) \mu(\mathrm{d} t)
$$

for $s \in \mathcal{C}$ and $\mu \in \mathcal{M}$ (Riesz's Representation Theorem).

Definition A (signed, finite) Borel measure on $[0, T]$ is a countably additive real-valued set function on the Borel sigma-algebra; i.e., it is a $\mu: \mathfrak{B}[0, T] \rightarrow \mathbb{R}$ with $\mu\left(\bigcup_{m=1}^{\infty} B_{m}\right)$ $=\sum_{m=1}^{\infty} \mu\left(B_{m}\right)$ for every sequence of pairwise disjoint sets $B_{m} \in \mathfrak{B}[0, T]$. The vector space of all Borel measures on $[0, T]$ is denoted by $\mathcal{M}[0, T]$. For some of its uses in optimization, see, e.g., [2] and [18]. 
The available capacities are taken to equal the installed capacities, and therefore to be constant over the cycle. This does play a part in some of the main results, including the determinacy of rental values (Lemma 8 and Theorem 9). However, to take full advantage of sensitivity analysis, the constant existing capacities $k$ are perturbed with increments $\Delta k$ which are periodic functions of time; this is further explained in Section 5 . (The notation $\Delta k$, etc., is always to be interpreted as a single symbol meaning "an increment to $k$ ".)

On the assumption of constant hydro capacities

$$
k_{\mathrm{H}}=\left(k_{\mathrm{St}}, k_{\mathrm{Tu}}\right)
$$

the long-run (LR) production set of the hydro technique is the convex cone ${ }^{11}$

$$
\begin{aligned}
\mathbb{Y}_{\mathrm{H}}:=\left\{\left(y,-k_{\mathrm{H}},-e\right) \in\right. & L_{+}^{\infty}[0, T] \times \mathbb{R}_{-}^{2} \times L_{-}^{\infty}[0, T]: 0 \leq y \leq k_{\mathrm{Tu}} \text { and } \\
& \left.\exists s \exists \sigma \in[0, e]\left(\dot{s}=-y+e-\sigma, s(0)=s(T) \text { and } 0 \leq s \leq k_{\mathrm{St}}\right)\right\} .
\end{aligned}
$$

The formulation imposes the periodicity or balance constraint $s(T)=s(0)$, but this stock level at the beginning or end of a cycle is taken to be a costless decision variable. In other words, when it is first commissioned, the reservoir comes filled up to any required level at no extra cost, but its periodic operation thereafter is taken to be a technological constraint. ${ }^{12}$

Thus our formulation is purely cyclic, unlike that of Koopmans [20, p. 196], which has separate initial and final stock levels (as data, not decisions). Another "purely cyclic" alternative is to regard the initial and final stock levels as equal but given, not chosen: this is so in, e.g., [3]. But if this level is arbitrary, there is no good reason to keep it for the start of the next period, and it would not be optimal to do so: generally, with a multi-period planning horizon, it would be best to build the level of $s(0)=s(T)$ up, to a long-term "turnpike" optimum (running a surplus or deficit over a number of cycles), and then keep it approximately the same. Such a long-run solution is likely to be approximated better by optimizing over $s(0)=s(T)$ than by fixing it at a particular level.

\section{$3 \quad$ Heuristics for valuation of water and capacities}

To start with, assume that not only the market price of electricity, $p(t)$, but also the shadow price of water, $\psi(t)$, is known. ${ }^{13}$ Then the operating decisions can be decentralized within the hydro plant, with the reservoir "buying" water at the price $\psi(t)$ from the river and "selling" it to the turbine, which in turn sells the generated electricity at the market price $p(t)$ outside the

\footnotetext{
${ }^{11}$ The constraint on spillage $\sigma \leq e$ is actually never binding: see Section 10 .

${ }^{12}$ It is easy to add a given price $q>0$ for the use of an initial stock (keeping the periodicity constraint), and to give an upper bound on q. The analysis remains the same except that $\psi(0)-\psi(T)$ equals q instead of zero, if $p(0)=p(T)$. And, since the minimum time required to build the stock up to any sustainable level is never more than one cycle (and is usually less), a reasonable q times the optimum of $s(0)=s(T)$ cannot exceed the interest on a period's operating profit as calculated from the present model (in which $\mathbf{q}=0$ ).

${ }^{13}$ When $\psi$ is formally introduced, as the Lagrange multiplier paired with the parameter $e$, it is by definition the price of the inflowing water. However, it must equal the price of water stored for hydro generation because, by assumption, there is no alternative use. This is why the inflow's price cannot exceed that of the stock. The reverse inequality is obvious.
} 
plant. The short-run profit maximization separates into problems with obvious solutions, one for each kind of capacity. The maximum profits of the reservoir and the turbine, $\Pi^{\mathrm{St}}\left(\psi, k_{\mathrm{St}}\right)$ and $\Pi^{\mathrm{Tu}}\left(p-\psi, k_{\mathrm{Tu}}\right)$, are both linear in $k$. A unit turbine can earn the profit flow $(p-\psi)^{+}$, which is the nonnegative part of $p-\psi$, by generating when $p(t)>\psi(t)$. The profit is earned only at the times of full capacity utilization, since the optimum output is $y_{\mathrm{H}}(t)=k_{\mathrm{Tu}}$ when $p(t)>\psi(t)$ : see Figures 1a and 1b. In total over the cycle, the value of a unit turbine is therefore $\Pi^{\mathrm{Tu}} / k_{\mathrm{Tu}}=\int_{0}^{T}(p(t)-\psi(t))^{+} \mathrm{d} t$. As for the reservoir, a unit can earn a profit of $\psi(\bar{\tau})-\psi(\underline{\tau})$ by buying stock at time $\underline{\tau}$ and selling it at a later time $\bar{\tau}$ when $\psi(\bar{\tau})>\psi(\underline{\tau})$. So the value of a unit reservoir is the sum of all shadow price rises in a cycle. In precise terms: if $\psi(T) \geq \psi(0)$, then $\Pi^{\mathrm{St}} / k_{\mathrm{St}}=\operatorname{Var}^{+}(\psi)$, which denotes the total positive variation (a.k.a. upper variation) of $\psi$, i.e., the supremum of $\sum_{m}\left(\psi\left(\bar{\tau}_{m}\right)-\psi\left(\underline{\tau}_{m}\right)\right)^{+}$over all finite sets of pairwise disjoint subintervals $\left(\underline{\tau}_{m}, \bar{\tau}_{m}\right)$ of $(0, T)$. (For a discussion of $\operatorname{Var}^{+}$see, e.g., [8, Section 8.1].)

If $\psi(T)<\psi(0)$, the reservoir should start the cycle full, and refill towards the end of the cycle. This brings an extra profit of $\psi(0)-\psi(T)$, so in general the unit rent is the cyclic positive variation

$$
\operatorname{Var}_{\mathrm{c}}^{+}(\psi):=\operatorname{Var}^{+}(\psi)+(\psi(0)-\psi(T))^{+} .
$$

Later it is shown that actually $\psi(0)=\psi(T)$ if $p(0)=p(T)$ and $p \in \mathcal{C}[0, T]$.

The maximum operating profit of the whole hydro plant $\left(\Pi_{\mathrm{SR}}^{\mathrm{H}}\right)$ is, however, a function not of $\psi$ but of the problem's parameters $\left(p, k_{\mathrm{H}}, e\right)$ alone: $\psi$ is an auxiliary function which must eventually be given in terms of $\left(p, k_{\mathrm{H}}, e\right)$. Then $\partial \Pi_{\mathrm{SR}}^{\mathrm{H}} / \partial k_{\mathrm{St}}$ and $\partial \Pi_{\mathrm{SR}}^{\mathrm{H}} / \partial k_{\mathrm{Tu}}$ can be obtained by substituting the correct $\psi$ into the expressions $\operatorname{Var}_{\mathrm{c}}^{+}(\psi)$ and $\int_{0}^{T}(p(t)-\psi(t))^{+} \mathrm{d} t$.

The correct value, $\hat{\psi}$, is the water price function that minimizes the value of the hydro plant's fixed resources $\left(k_{\mathrm{H}}, e\right)$. So, given a TOU electricity price $p$, one can find $\hat{\psi}$ by unconstrained minimization of

$$
k_{\mathrm{St}} \operatorname{Var}_{\mathrm{c}}^{+}(\psi)+k_{\mathrm{Tu}} \int_{0}^{T}(p(t)-\psi(t))^{+} \mathrm{d} t+\int_{0}^{T} \psi(t) e(t) \mathrm{d} t
$$

over $\psi$, an arbitrary bounded-variation function on $(0, T)$.

In the case of $k_{\mathrm{Tu}}>e(t)>0$ for every $t$, the sum of the two integrals in (5) has a minimum at (and only at) $\psi=p .{ }^{14}$ Therefore the main feature of this programme is the trade-off between minimizing the variation (which on its own would require setting $\psi$ at a constant value) and minimizing the integrals (which on its own would require setting $\psi$ equal to $p$ ). From this trade-off it is clear to what extent the local peaks of $p$ should be "shaved off" and the troughs "filled in" to obtain the optimum shadow price $\hat{\psi}$ - at least in the case that the market price $p$ is piecewise strictly monotone and $k_{\mathrm{Tu}}>e>0$ at all times. (An extension dispensing with the upper bound on $e$ is sketched in Section 10.) The solution, presented graphically in Figure 1a, is determined by constancy intervals of $\hat{\psi}$, on each of which $p(t)-\hat{\psi}$ has a constant sign. If

\footnotetext{
${ }^{14}$ This is proved by subdifferentiating, w.r.t. $\psi$, the two terms $V_{\mathrm{Tu}}(\psi):=k_{\mathrm{Tu}} \int_{0}^{T}(p-\psi)^{+} \mathrm{d} t$ and $V_{\mathrm{Ri}}(\psi):=$ $\int_{0}^{T} \psi e \mathrm{~d} t$. For a rigorous proof, consider $V=V_{\mathrm{Tu}}+V_{\mathrm{Ri}}$ as a convex and $\|\cdot\|_{1}$-continuous function on $L^{1}[0, T]$. It has a minimum at a $\psi$ if and only if $0 \in \partial V(\psi) \subset L^{\infty}$ (i.e., the zero function belongs to the subdifferential, a.k.a. the set of all subgradients, of $V$ at $\psi$ ). And $g \in \partial V_{\mathrm{Tu}}(\psi)$ if and only if: $g=k_{\mathrm{Tu}}$ a.e. on $\{t: \psi<p\}$, $k_{\mathrm{Tu}} \geq g \geq 0$ a.e. on $\{t: \psi=p\}$, and $g=0$ a.e. on $\{t: \psi>p\}$. Also, $\nabla V_{\mathrm{Ri}}=e$. Since $k_{\mathrm{Tu}}>e>0$ a.e., it follows that $0 \in \partial V(\psi)$ if and only if $\psi=p$ a.e.
} 
$k_{\mathrm{St}} / \operatorname{Min}(e)$ and $k_{\mathrm{St}} /\left(k_{\mathrm{Tu}}-\operatorname{Max}(e)\right)$, upper bounds on the times needed to fill up and to empty the reservoir, are sufficiently short, then the constancy intervals do not abut. Around a trough of $p$, there is an interval $(\underline{t}, \bar{t})$ characterized by

$$
k_{\mathrm{St}}=\int_{\underline{t}}^{\bar{t}} e(t) \mathrm{d} t
$$

on which $p(t)<\hat{\psi}$ throughout. Around a local peak of $p$, there is an interval $(\underline{t}, \bar{t})$ characterized by

$$
k_{\mathrm{St}}=\int_{\underline{t}}^{\bar{t}}\left(k_{\mathrm{Tu}}-e(t)\right) \mathrm{d} t
$$

on which $p(t)>\hat{\psi}$ throughout. These are the first-order conditions (FOCs) for the dual optimum: (6) or (7) is obtained by equating to zero the increment in the minimand (5) that results from shifting the constant value of $\psi$ by an infinitesimal unit, on an interval around a peak or a trough of $p{ }^{15}$ These conditions make it feasible to produce the "bang-coast-bang" output (viz., $y(t)=k_{\text {Tu }}$ when $\hat{\psi}(t)<p(t), y(t)=e(t)$ when $\hat{\psi}(t)=p(t)$, and $y(t)=0$ when $\hat{\psi}>p)$ : the reservoir goes alternately from empty to full and vice versa (Figures $1 \mathrm{~b}$ and $1 \mathrm{c}$ ). This is the optimal output.

The same marginal calculation for the dual problem also shows that an optimum $\psi$ can be nonunique if $p$ is discontinuous. Suppose, for example, that $p$ jumps at the beginning, and drops at the end, of an interval $A=(\underline{t}, \bar{t})$ meeting (7) and the condition

$$
\max \{p(\underline{t}-), p(\bar{t}+)\}<\min \{p(\underline{t}+), p(\bar{t}-)\}=\inf _{t \in A} p(t) .
$$

Just before $\underline{t}$ and just after $\bar{t}$, an optimal $\psi$ equals $p$, i.e., $\psi(\underline{t}-)=p(\underline{t}-)$ and $\psi(\bar{t}+)=p(\bar{t}+)$. Inside $A, p>\psi=$ const.; but an optimal constant value of $\psi$ on $A$ can be anywhere between the two unequal terms of (8): the jump and the drop of $p$ create an "indifference zone" for $\psi_{\mid A}$. Figure 2 shows this when $p(\bar{t}+) \leq p(\underline{t}-)<p(\underline{t}+) \leq p(\bar{t}-)$ so $p(\underline{t}-) \leq \psi_{\mid A} \leq p(\underline{t}+)$. Different values from this range divide the same total rent differently between the three fixed inputs: the jump $\mathrm{d} \psi\{\underline{t}\}:=\psi(\underline{t}+)-\psi(\underline{t}-)$ can be any fraction of $p(\underline{t}+)-p(\underline{t}-)$, and it is an indeterminate contribution to the reservoir's unit rent. The interval's contribution to the turbine's rent, $\int_{A}(p(t)-\psi) \mathrm{d} t$, is similarly indeterminate (since it depends on the arbitrary choice of $\psi(\underline{t}+)$, which fixes the constant value of $\psi$ on $A$ ). And the indeterminate $\psi_{\mid A}$ itself is the river's unit rent, on $A .^{16}$

Conversely, given a continuous $p$, there is a unique optimum, $\hat{\psi}$ (Lemma 8). Therefore the gradient $\nabla_{k, e} \Pi$ exists, and $\nabla_{e} \Pi=\hat{\psi}$ (Theorem 9). The directional derivative of $\Pi$ with respect to the capacities and the inflow is then a linear function of their increments, i.e.,

$$
\mathrm{D}_{\mathrm{SR}}^{\mathrm{H}}\left(\Delta k_{\mathrm{St}}, \Delta k_{\mathrm{Tu}}, \Delta e\right)=\frac{\partial \Pi_{\mathrm{SR}}^{\mathrm{H}}}{\partial k_{\mathrm{St}}} \Delta k_{\mathrm{St}}+\frac{\partial \Pi_{\mathrm{SR}}^{\mathrm{H}}}{\partial k_{\mathrm{Tu}}} \Delta k_{\mathrm{Tu}}+\left\langle\nabla_{e} \Pi_{\mathrm{SR}}^{\mathrm{H}}, \Delta e\right\rangle
$$

\footnotetext{
${ }^{15}$ Matters complicate when, for relatively large $k_{\mathrm{St}}$, the neighbouring intervals of water collection and of discharge abut; but a similar optimality rule applies to such clusters: see [11].

${ }^{16}$ The case of $p$ dropping at the beginning, and jumping at the end, of an interval $A=(\underline{t}, \bar{t})$ that meets Condition (6) is similar, except that the turbine's rent on $A$ is zero (since $p<\psi_{\mid A}$ ).
} 


$$
=\Delta k_{\mathrm{St}} \operatorname{Var}_{\mathrm{c}}^{+}(\hat{\psi})+\Delta k_{\mathrm{Tu}} \int_{0}^{T}(p(t)-\hat{\psi}(t))^{+} \mathrm{d} t+\int_{0}^{T} \hat{\psi}(t) \Delta e(t) \mathrm{d} t
$$

(with all the derivatives and $\hat{\psi}$ evaluated at the given $k_{\mathrm{H}}$ and $e$ ). So the profit-imputed value of investment is (jointly) additive in all the increments (unlike Koopmans's cost-imputed incremental value, which is calculated from a multi-valued subdifferential $\partial_{k, e} C$ of the short-run cost, instead of our single-valued gradient $\left.\nabla_{k, e} \Pi\right)$.

\section{The linear programme of profit-maximizing plant operation}

For a hydro plant with storage and turbine capacities $k_{\mathrm{H}}=\left(k_{\mathrm{St}}, k_{\mathrm{Tu}}\right)$, and with a river inflow $e$, the operation problem is to maximize the value of electricity output $y$, at a given TOU price represented by an integrable function $p$ on $[0, T]$, subject to the technological constraints in (3), i.e.,

$$
\begin{aligned}
& \text { Given }\left(p, k_{\mathrm{H}}, e\right) \in L^{1}[0, T] \times \mathbb{R}^{2} \times L^{\infty}[0, T] \\
& \text { maximize }\langle p, y\rangle \text { over } y \in L^{\infty}[0, T] \\
& \text { subject to: }\left(y,-k_{\mathrm{H}},-e\right) \in Y_{\mathrm{H}} \text { defined by }(3) \text {. }
\end{aligned}
$$

The plant operation problem is next formulated as an LP by expanding the technological constraint (12) into turbine constraints on the output rate, and reservoir constraints and a balance constraint on the water stock. To exclude spillage (i.e., make it unnecessary and unprofitable), we assume that the river inflow rate never exceeds the turbine capacity (i.e., that $e \leq k_{\mathrm{Tu}}$ until Section 10), and that the electricity price is strictly positive at all times (i.e., $\left.p \in L_{++}^{1}[0, T]\right)$. With the constants $k_{\mathrm{St}}$ and $k_{\mathrm{Tu}}$ viewed as special cases of cyclically varying functions, the primal LP of plant operation is:

$$
\begin{aligned}
& \text { Given }\left(p ; k_{\mathrm{St}}, k_{\mathrm{Tu}} ; e\right) \in L_{++}^{1} \times \mathbb{R}_{+}^{2} \times L_{+}^{\infty} \subset L_{++}^{1} \times\left(\mathcal{C}_{+} \times L_{+}^{\infty}\right) \times L_{+}^{\infty} \text { with } k_{\mathrm{Tu}} \geq e \\
& \text { maximize } \int_{0}^{T} p(t) y(t) d t \text { over } y \in L^{\infty}[0, T] \text { and } s_{0} \in \mathbb{R} \\
& \text { subject to: } 0 \leq y(t) \leq k_{\mathrm{Tu}} \quad \text { for a.e. } t \\
& \qquad \int_{0}^{T}(y(t)-e(t)) \mathrm{d} t=0 \\
& 0 \leq s_{0}-\int_{0}^{t}(y(\tau)-e(\tau)) \mathrm{d} \tau \leq k_{\mathrm{St}} \quad \text { for every } t
\end{aligned}
$$

where $s_{0}$ means the initial stock (and $y$ the output), and so the associated stock trajectory is $s(t)=s_{0}-\int_{0}^{t}(y-e)(\tau) \mathrm{d} \tau$ with $s(0)=s_{0}$ of course. ${ }^{17}$

Notation The optimal value of (10)-(12) or (13)-(17) is the (maximum) operating profit of the hydro plant, denoted by $\Pi_{\mathrm{SR}}^{\mathrm{H}}\left(p, k_{\mathrm{H}}, e\right)$. The (optimal) solution set of (10)-(12) is

\footnotetext{
${ }^{17}$ There is a syntactical reason for using $s_{0}$, understood as one symbol, to mean the initial stock when it is a decision variable, as in (14): $s(0)$ is a complex, "parse-able" expression which should not serve as a variable's name (a similar distinction is usually made in initial value problems).
} 
$\hat{Y}_{\mathrm{H}}\left(p, k_{\mathrm{H}}, e\right)$, occasionally abbreviated to $\hat{Y}$. The corresponding lowercase notation $\hat{y}$ is used only when the solution is known to be unique. Also, the space $L^{1}$ appearing in (10) consists of all functions integrable with respect to (w.r.t.) meas, the Lebesgue measure. The integral $\int_{0}^{T} p(t) y(t) \mathrm{d} t$ is also written as $\langle p, y\rangle .{ }^{18}$ The condition $p>0$ a.e. on $[0, T]$ is also written as $p \gg 0$, or as $p \in L_{++}^{1}$.

The two formulations of the operation problem are equivalent in the sense that $y$ solves (10)-(12) if and only if $y$ together with some $s_{0}$ solves (13)-(17) - in which case $y$ together with the specific value

$$
\underline{s}_{0, y}:=\max _{t \in[0, T]}\left(\int_{0}^{t}(y(\tau)-e(\tau)) \mathrm{d} \tau\right)
$$

is a solution: $\underline{s}_{0, y}$ is the lowest initial stock required for $s(t)$ never to fall below 0 . (Unless there is spare storage capacity, this is actually the only feasible value for $s_{0}$, given $y$.) One can therefore restrict attention to points $\left(y, s_{0}\right)$ with $s_{0}=\underline{s}_{0, y}$; and so the stock trajectory associated with a hydro output $y$ is

$$
s(t)=\underline{s}_{0, y}-\int_{0}^{t}(y(\tau)-e(\tau)) \mathrm{d} \tau .
$$

The dual programme, introduced next, serves the purposes of characterizing optimal operation and calculating the marginal values of the capacities and the inflow. To ensure that the dual has a solution of the kind sketched in Section 3, for the most part it is assumed from here on that

$$
k_{\mathrm{Tu}}>\operatorname{EssSup}(e) \geq \operatorname{EssInf}(e)>0 \quad \text { and } \quad k_{\mathrm{St}}>0 .
$$

This means that the "pure coasting" policy (i.e., $y=e$ with $\sigma=0$ ) is feasible and, furthermore, that it verifies Slater's Condition for the primal. ${ }^{19}$

\section{Fixed-input valuation as the dual linear programme}

As is set out in, e.g., [24], the dual to a convex programme depends on the choice of perturbations for the primal parameters. A choice of admissible perturbations determines the structure of the dual variables (a.k.a. Lagrange multipliers) to be paired with the parameter increments. Therefore, the dual programme depends not only on the particular values of the primal parameters, but also on the vector space of parameter increments or perturbations. This "ambient" space for the given parameter point can be chosen to suit one's purpose.

In the case of (13)-(17), the programme contains a separate set of capacity constraints for each time $t$ - so, by considering a separate increment $\Delta k_{\mathrm{H}}(t)$ for each $t$, one can impute an

\footnotetext{
${ }^{18}$ The revenue flow is not discounted within the cycle because all the prices are in present-value terms. The same applies to the shadow stock prices $\psi$, to be introduced formally in (27); so the rises of $\psi$ give stock appreciation net of the interest on its value.

${ }^{19}$ This standard constraint qualification for CPs is, in the infinite-dimensional case, useful with LPs as well. Without it, the primal and dual values may be different, or there may be no dual optimum. For example, if $p \in L^{1}, k_{\mathrm{Tu}}>e>0$ but $k_{\mathrm{St}}=0$, then the primal and dual values are the same (viz., $\int p e \mathrm{~d} t$ ), but an (exact) dual optimum exists only if $p \in \mathrm{BV}$ (in which case the optimal stock price is $\hat{\psi}=p$ ). See also [2, p. 31.].
} 
instantaneous value, $\kappa_{\mathrm{H}}(t)$, to capacity services at each time $t$. In other words, not only their total value, but also its distribution over the cycle can be determined. Even if the existing capacities $k_{\mathrm{H}}$ are actually constant, it is useful to consider the cyclically varying increments $\Delta k_{\mathrm{H}}$ because this gives a marginal interpretation to the time-dependent Lagrange multipliers for the capacity constraints: denoted by $\kappa_{\mathrm{H}}=\left(\kappa_{\mathrm{St}}, \kappa_{\mathrm{Tu}}\right)$, these are the unit values of the capacities' services at any particular time. As part of the "variation of constants", we also consider a varying increment $\Delta n_{\mathrm{St}}(t)$ to the zero floor for the water stock in (17), and a varying increment $\Delta n_{\mathrm{Tu}}(t)$ to the zero floor for the turbine output rate in (15). This gives a marginal interpretation to the time-dependent Lagrange multipliers for the nonnegativity constraints: denoted by $\nu_{\mathrm{H}}=\left(\nu_{\mathrm{St}}, \nu_{\mathrm{Tu}}\right)$, these are the unit values of lowering the "floors" at any time. Finally, a scalar $\Delta \zeta$ is an increment to the zero on the r.h.s. of (16); this can be thought of as the quantity of water taken to be available for topping up the reservoir between cycles. Its multiplier, a scalar $\lambda$, is the marginal value of water at the beginning (or end) of cycle. All the multipliers $\left(\kappa_{\mathrm{H}}, \nu_{\mathrm{H}}\right.$ and $\left.\lambda\right)$ are terms of the TOU electricity price $p$ in its decomposition (26) $-(27)$ below, which is a part of the dual programme's constraints.

The short-run profit maximization problem (13)-(17) is thus embedded in the family of perturbed programmes obtained by adding an arbitrary cyclically varying increment $\left(\Delta k_{\mathrm{St}}\right.$, $\left.\Delta n_{\mathrm{St}}, \Delta k_{\mathrm{Tu}}, \Delta n_{\mathrm{Tu}}, \Delta e\right)$ and a scalar $\Delta \zeta \in \mathbb{R}$ to the particular parameter point consisting of the constants $\left(k_{\mathrm{St}}, 0, k_{\mathrm{Tu}}, 0, e\right)$ and 0 . The function spaces for the resource increments, indicated already in (13), are: $\mathcal{C}[0, T]$ for $\Delta k_{\mathrm{St}}$ and $\Delta n_{\mathrm{St}}$, and $L^{\infty}[0, T]$ for $\Delta k_{\mathrm{Tu}}$ and $\Delta n_{\mathrm{Tu}}$. These are paired with $\mathcal{M}[0, T]$ and $L^{1}[0, T]$ as spaces for the shadow prices, i.e., Lagrange multipliers. (The pairing of $L^{\infty}$ with its norm-dual $L^{\infty *}$, instead of the smaller space $L^{1}$ is also needed, but only in proving the dual's solubility: both $\kappa_{\mathrm{Tu}}$ and $\nu_{\mathrm{Tu}}$ are actually in $L^{1}$, although $\kappa_{\mathrm{Tu}} \in L^{1}$ only because $p \in L^{1}$ instead of $L^{\infty *}$.)

In other words, the marginal value of the storage capacity services on an interval $A \subset[0, T]$ is given by a measure $\kappa_{\mathrm{St}}(A)$; this is the incremental operating profit from the availability on $A$ of an extra unit of the reservoir. Another measure, $\nu_{\mathrm{St}}(A)$, gives the incremental profit from lowering the stock floor by a unit, on $A$. The marginal value of the turbine capacity services, on $A$, is the Lebesgue integral of a function $\kappa_{\mathrm{Tu}} \in L^{1}$. The value of lowering the turbine output floor by a unit is the integral of another function, $\nu_{\mathrm{Tu}} \in L^{1}$.

Thus the complete shadow-price system $\left(\kappa_{\mathrm{St}}, \nu_{\mathrm{St}} ; \kappa_{\mathrm{Tu}}, \nu_{\mathrm{Tu}} ; \psi, \lambda\right)$ values all the resource increments $\left(\Delta k_{\mathrm{St}},-\Delta n_{\mathrm{St}} ; \Delta k_{\mathrm{Tu}},-\Delta n_{\mathrm{Tu}} ; \Delta e, \Delta \zeta\right)$. Of course, it also values the particular resource bundle $\left(k_{\mathrm{St}}, 0 ; k_{\mathrm{Tu}}, 0 ; e, 0\right)$ that represents the plant itself-and the dual to the operation programme (13)-(17) is to minimize the plant's value by an admissible choice of the shadow prices. The main dual constraints (26)-(27) decompose the electricity price $p$ into the sum of: the turbine capacity charge $\kappa_{\mathrm{Tu}}$, minus the value of the output floor $\nu_{\mathrm{Tu}}$, and the shadow price of water $\psi$. The water price is the sum of: the initial price $\lambda$, the cumulative of reservoir capacity charges $\kappa_{\mathrm{St}}$, and the cumulative of $-\nu_{\mathrm{St}}$. This is spelt out next.

Theorem 1 (Fixed-input value minimization as the dual) The dual of the linear programme (13)-(17), relative to the specified perturbation and the pairing of the parameter spaces $\mathcal{C}$ and $L^{\infty}$ with $\mathcal{M}$ and $L^{1}$ respectively, is:

$$
\text { Given }\left(p ; k_{\mathrm{H}}, e\right) \text { as in }(13)
$$




$$
\begin{aligned}
& \text { minimize }_{k_{\mathrm{St}}} \int_{[0, T]} \kappa_{\mathrm{St}}(\mathrm{d} t)+k_{\mathrm{Tu}} \int_{0}^{T} \kappa_{\mathrm{Tu}}(t) \mathrm{d} t+\int_{0}^{T} \psi(t) e(t) \mathrm{d} t \\
& \text { over } \lambda \in \mathbb{R}, \psi \in L^{1}[0, T] \text { and }\left(\kappa_{\mathrm{St}}, \nu_{\mathrm{St}} ; \kappa_{\mathrm{Tu}}, \nu_{\mathrm{Tu}}\right) \in \mathcal{M}[0, T] \times \mathcal{M}[0, T] \times L^{1}[0, T] \times L^{1}[0, T] \\
& \text { subject to: }\left(\kappa_{\mathrm{St}}, \nu_{\mathrm{St}} ; \kappa_{\mathrm{Tu}}, \nu_{\mathrm{Tu}}\right) \geq 0 \\
& \kappa_{\mathrm{St}}[0, T]=\nu_{\mathrm{St}}[0, T] \\
& \quad p=\psi+\kappa_{\mathrm{Tu}}-\nu_{\mathrm{Tu}} \\
& \psi=\lambda+\left(\kappa_{\mathrm{St}}-\nu_{\mathrm{St}}\right)[0, \cdot] .
\end{aligned}
$$

Remark 2 Under (20), any solution to (21)-(27) has the disjointness properties that

$$
\kappa_{\phi} \wedge \nu_{\phi}=0 \quad \text { for } \phi=T u, S t \quad \text { and } \quad \kappa_{\mathrm{St}}\{0, T\} \wedge \nu_{\mathrm{St}}\{0, T\}=0
$$

i.e., it is not optimal for the dual variables to overlap and partly cancel each other out. ${ }^{20}$

\section{Conditions for optimal operation and valuation}

The dual programme (21)-(27) has a solution, (in which $\psi \in \mathrm{BV}(0, T)$ by $(27)$ and $\nu_{\mathrm{Tu}}$ and $\kappa_{\text {Tu }}$ are in $L^{1}$ because $p \in L^{1}$, whilst $\kappa_{\text {St }}$ and $\nu_{\text {St }}$ are in $\left.\mathcal{M}\right)$. The primal and dual optima are characterized by the Kuhn-Tucker Conditions, which for LPs reduce to feasibility and complementary slackness. Spelt out next, these conditions are later used to determine plant operation in terms of the water price, and to establish that this shadow price is unique.

Proposition 3 (Dual solubility and optimality conditions) Assume (20). Then:

1. The fixed-input value minimization programme (21)-(27) has an (optimal) solution

$$
\left(\kappa_{\mathrm{St}}, \nu_{\mathrm{St}} ; \kappa_{\mathrm{Tu}}, \nu_{\mathrm{Tu}} ; \psi, \lambda\right) \in \mathcal{M}[0, T] \times \mathcal{M}[0, T] \times L^{1} \times L^{1} \times \mathrm{BV}(0, T) \times \mathbb{R} .
$$

The programme's value is finite and equal to the short-run profit $\Pi_{\mathrm{SR}}^{\mathrm{H}}\left(p, k_{\mathrm{H}}, e\right)$, the optimal value of (13)-(17).

2. Points $\left(y, \underline{s}_{0, y}\right) \in L^{\infty} \times \mathbb{R}$ and $\left(\kappa_{\mathrm{St}}, \nu_{\mathrm{St}} ; \kappa_{\mathrm{Tu}}, \nu_{\mathrm{Tu}} ; \psi, \lambda\right)$ are optimal solutions to, respectively, the primal (13)-(17) and the dual (21)-(27) if and only if:

(a) $\left(y, \underline{s}_{0, y}\right)$ and $\left(\kappa_{\mathrm{St}}, \nu_{\mathrm{St}} ; \kappa_{\mathrm{Tu}}, \nu_{\mathrm{Tu}} ; \psi, \lambda\right)$ are feasible, i.e., satisfy (15)-(17) and (24)(27).

(b) The measure $\kappa_{\mathrm{St}}$ is concentrated on $\left\{t \in[0, T]: s(t)=k_{\mathrm{St}}\right\}$, whilst $\nu_{\mathrm{St}}$ is concentrated on $\{t: s(t)=0\}$, where $s$ is given by (18)-(19).

(c) The function $\kappa_{\mathrm{Tu}}$ vanishes a.e. outside of $\left\{t: y(t)=k_{\mathrm{Tu}}\right\}$, whilst $\nu_{\mathrm{Tu}}$ vanishes outside of $\left\{t: y_{\mathrm{Tu}}(t)=0\right\}$.

\footnotetext{
${ }^{20}$ For $\phi=$ St, this means that $\kappa_{\mathrm{St}}$ and $\nu_{\mathrm{St}}$ are disjoint as measures on the circle obtained from the interval $[0, T]$, and not only on $[0, T]$ itself.
} 


\section{Shadow pricing of water as the dual problem}

The dual problem can be transformed into one of unconstrained minimization over $\psi \in \mathrm{BV}(0, T)$ by using the dual constraints (25)-(27) and the disjointness condition (28) to eliminate the other dual variables $\left(\kappa_{\mathrm{St}}, \nu_{\mathrm{St}} ; \kappa_{\mathrm{Tu}}, \nu_{\mathrm{Tu}} ; \lambda\right)$.

Notation The space $\mathrm{BV}(0, T)$ consists of all functions $\psi$ of bounded variation on $(0, T)$ with $\psi(t)$ lying between the left and right limits, $\psi(t-)=\lim _{\tau / t} \psi(\tau)$ and $\psi(t+)$ $=\lim _{\tau \backslash t} \psi(\tau) .{ }^{21} \mathrm{~A} \psi \in \mathrm{BV}(0, T)$ is extended by continuity to $[0, T]$; i.e., $\psi(0):=\psi(0+)$ and $\psi(T):=\psi(T-)$. The cyclic positive variation of $\psi$ is defined by (4).

If finite numbers $\psi(0-)$ and $\psi(T+)$ are additionally specified, then $\psi \in \mathrm{BV}[0-, T+]$; and such a $\psi$ defines a measure on $[0, T]$ by

$$
\mathrm{d} \psi\left[t^{\prime}, t^{\prime \prime}\right]:=\psi\left(t^{\prime \prime}+\right)-\psi\left(t^{\prime}-\right)
$$

for $t^{\prime} \leq t^{\prime \prime}$. The integral of $s$ w.r.t. the measure $(\mathrm{d} \psi)^{+}$is also written as $\int s(\mathrm{~d} \psi)^{+}$. When $\psi(0-)=\psi(T+)$, the usual variation norm of $(\mathrm{d} \psi)^{+}$equals $\operatorname{Var}_{\mathrm{c}}^{+}(\psi)$.

It is convenient to set

$$
\psi(0-)=\psi(T+)=\lambda
$$

so that, from (27) and (25),

$$
\kappa_{\mathrm{St}}-\nu_{\mathrm{St}}=\mathrm{d} \psi \quad \text { on }[0, T] .
$$

From this, (24) and (28),

$$
\begin{gathered}
\kappa_{\mathrm{St}}=(\mathrm{d} \psi)^{+} \text {and } \nu_{\mathrm{St}}=(\mathrm{d} \psi)^{-} \\
\psi(0+) \wedge \psi(T-) \leq \lambda \leq \psi(0+) \vee \psi(T-) .
\end{gathered}
$$

i.e., $\lambda$ lies between $\psi(0+)$ and $\psi(T-)$. All choices of $\lambda$ from this range are equally good, i.e., contribute the same to (22). Lastly, from (24), (26) and (28),

$$
\kappa_{\mathrm{Tu}}=(p-\psi)^{+} \quad \text { and } \quad \nu_{\mathrm{Tu}}=(p-\psi)^{-} .
$$

Proposition 4 (Shadow pricing of water as the dual) Assume (20). The fixed-input value minimization programme (21)-(27) is then equivalent to the following convex programme:

$$
\begin{aligned}
& \text { Given }\left(p ; k_{\mathrm{H}}, e\right) \text { as in (13) } \\
& \text { minimize } k_{\mathrm{St}} \operatorname{Var}_{\mathrm{c}}^{+}(\psi)+k_{\mathrm{Tu}} \int_{0}^{T}(p-\psi)^{+} \mathrm{d} t+\int_{0}^{T} \psi(t) e(t) \mathrm{d} t \\
& \text { over } \psi \in \mathrm{BV}(0, T) \text {. }
\end{aligned}
$$

Notation The solution set for $(33)-(35)$ is denoted by $\hat{\Psi}\left(p, k_{\mathrm{H}}, e\right) \neq \emptyset$. Again, the corresponding lowercase notation $\hat{\psi}$ is used only when the dual solution is unique.

\footnotetext{
${ }^{21}$ The one-sided limits exist at every $t$ and are equal nearly everywhere (n.e.), i.e., everywhere except for a countable set. Specification of $\psi(t)$ between $\psi(t-)$ and $\psi(t+)$ is unnecessary.
} 
It is this formulation of the dual that leads to the idea of obtaining $\hat{\psi}$ by "levelling off" the local extremes of $p$ in the way described in Section 3. The insight can be developed into a specialized algorithm when $p$ is piecewise monotone. In this approach, the dual is tackled first, in the CP form (33)-(35), and the primal solution is found subsequently. (For comparison: the simplex and other methods for LPs find both solutions simultaneously.)

\section{Determination of hydro output}

The plant operation problem is soluble for every $p \in L^{1}[0, T]$.

Proposition 5 (Primal solubility) Assume that $k_{\mathrm{Tu}} \geq e \geq 0$. If $p \in L^{1}$, then the short-run profit-maximizing operation programme (13)-(17) has an (optimal) solution $\left(y, s_{0}\right)$. It follows that the problem (10)-(12) has a solution, i.e., $\hat{Y}\left(p, k_{\mathrm{H}}, e\right) \neq \emptyset$.

Once the dual is solved, so that an optimal $\psi$ is known, the operation problem largely reduces to maximization of instantaneous profits (as Part 2c of Proposition 3 shows). At each $t$ with $p(t) \neq \psi(t)$, the optimum output $y(t)$ is of the "bang-bang control" type, either $k_{\text {Tu }}$ or 0 . Any remaining part of an optimal $y$ is a "singular control", which arises at a time $t$ when the instantaneous optimum is multi-valued because $\psi(t)=p(t)$. This part can be determined on the assumption (36) that $p$ has no plateau: this ensures that $p(t)=\psi(t)$ only when the reservoir is either empty or full; and at those times the output rate must equal $e(t)$. See Figure 1.

Proposition 6 (Hydro output with plateau-less price) In addition to assuming (20) and that $p \in L_{++}^{1}[0, T]$, assume that $p$ has no plateau, i.e., that

$$
\forall \mathrm{p} \in \mathbb{R}_{+} \text {meas }\{t: p(t)=\mathrm{p}\}=0 .
$$

If $y \in \hat{Y}\left(p, k_{\mathrm{H}}, e\right)$ and $\psi \in \hat{\Psi}\left(p, k_{\mathrm{H}}, e\right)$, i.e., y solves (10)-(12) and $\psi$ solves (33)-(35), then

$$
y(t)=\left\{\begin{array}{ll}
k_{\mathrm{Tu}} & \text { if } p(t)>\psi(t) \\
e(t) & \text { if } p(t)=\psi(t) \\
0 & \text { if } p(t)<\psi(t)
\end{array} .\right.
$$

So (10)-(12) has a unique solution $\hat{y}\left(p, k_{\mathrm{H}}, e\right){ }^{22}$

\section{Marginal capacity values in terms of water price}

By definition, $\Pi_{\mathrm{SR}}^{\mathrm{H}}\left(p, k_{\mathrm{H}}, e\right)$ is the optimal value of the primal (operation) problem, $\max _{y}\langle p, y\rangle$. Since the dual and primal values are equal (Proposition 3), a dual (water-pricing) solution $\psi$ gives $\Pi$ as the total fixed-input value (the plant's total rent on the capacities and the river inflow); and it has the advantage of giving the marginal values $\nabla_{k, e} \Pi$ as well.

\footnotetext{
${ }^{22}$ Since $y$ is fully determined in terms of any optimal $\psi$, it is unique (even though $\psi$ may be nonunique unless $p \in \mathcal{C})$.
} 
Corollary 7 (Dual calculation of SR profit) Assume (20). Then, for each $\psi \in \hat{\Psi}\left(p, k_{\mathrm{H}}, e\right)$,

$$
\Pi_{\mathrm{SR}}^{\mathrm{H}}\left(p, k_{\mathrm{H}}, e\right)=k_{\mathrm{St}} \operatorname{Var}_{\mathrm{c}}^{+}(\psi)+k_{\mathrm{Tu}} \int_{0}^{T}(p(t)-\psi(t))^{+} \mathrm{d} t+\int_{0}^{T} \psi(t) e(t) \mathrm{d} t
$$

Furthermore, this sum equals

$$
\int_{0}^{T} \psi(t)(y(t)-e(t)) \mathrm{d} t+\int_{0}^{T}(p(t)-\psi(t)) y(t) \mathrm{d} t+\int_{0}^{T} \psi(t) e(t) \mathrm{d} t
$$

term-by-term, for every $y \in \hat{Y}\left(p, k_{\mathrm{H}}, e\right) .^{23}$

Since $\Pi$ is positively homogeneous of degree 1 (a.k.a. linearly homogeneous) in $(k, e),{ }^{24}$ Euler's Theorem shows that if $\Pi$ is differentiable in $k,{ }^{25}$ then

$$
\Pi_{\mathrm{SR}}^{\mathrm{H}}\left(p, k_{\mathrm{H}}, e\right)=k_{\mathrm{St}} \frac{\partial \Pi_{\mathrm{SR}}^{\mathrm{H}}}{\partial k_{\mathrm{St}}}+k_{\mathrm{Tu}} \frac{\partial \Pi_{\mathrm{SR}}^{\mathrm{H}}}{\partial k_{\mathrm{Tu}}}+\left\langle\nabla_{e} \Pi_{\mathrm{SR}}^{\mathrm{H}}, e\right\rangle .
$$

A comparison with (38) suggests that if there is a unique optimal $\psi$, then the partial derivatives of $\Pi$ do exist and equal the coefficients of $k_{\mathrm{St}}, k_{\mathrm{Tu}}$ and $e$ in (38); formally this follows from (27) and the marginal interpretation of $\kappa_{\mathrm{St}}, \kappa_{\mathrm{Tu}}$ and $\psi$ (spelt out in the Proof of Theorem 9). And the optimal stock price $\psi$ is indeed unique if $p$, the TOU price of the good, is continuous over time.

Lemma 8 (Water price uniqueness and continuity) In addition to (20), assume that $p \in$ $\mathcal{C}_{++}[0, T]$. Then the dual (33)-(35) has a unique (optimal) solution $\hat{\psi}\left(p, k_{\mathrm{H}}, e\right)$, which belongs to $\mathcal{C}_{++}[0, T]$. If additionally $p(0)=p(T)$, then also $\hat{\psi}(0)=\hat{\psi}(T)$.

Theorem 9 (Efficiency rents of a hydro plant) Assume that $p \in \mathcal{C}_{++}[0, T]$. Then the operating profit of a hydro plant-i.e., the value of the primal problem (10)-(12)—is differentiable with respect to the water inflow function (e) and the capacities (of the reservoir and the turbine, $\left.k_{\mathrm{H}}=\left(k_{\mathrm{St}}, k_{\mathrm{Tu}}\right)\right)$, at every $\left(k_{\mathrm{H}}, e\right)$ satisfying (20). The derivatives defining the unit rents are given by the formulae

$$
\begin{aligned}
\frac{\partial \Pi_{\mathrm{SR}}^{\mathrm{H}}}{\partial k_{\mathrm{St}}}\left(p, k_{\mathrm{H}}, e\right) & =\operatorname{Var}_{\mathrm{c}}^{+}\left(\hat{\psi}\left(p, k_{\mathrm{H}}, e\right)\right) \\
\frac{\partial \Pi_{\mathrm{SR}}^{\mathrm{H}}}{\partial k_{\mathrm{Tu}}}\left(p, k_{\mathrm{H}}, e\right) & =\int_{0}^{T}\left(p(t)-\hat{\psi}\left(p, k_{\mathrm{H}}, e\right)(t)\right)^{+} \mathrm{d} t \\
\nabla_{e} \Pi_{\mathrm{SR}}^{\mathrm{H}} & \left(p, k_{\mathrm{H}}, e\right)=\hat{\psi}\left(p, k_{\mathrm{H}}, e\right)
\end{aligned}
$$

in which $\hat{\psi}$ is the unique solution to the dual problem (33)-(35) of water pricing.

\footnotetext{
${ }^{23}$ This shows that the values of the fixed inputs are equal to their profits $-\langle\psi, y-e\rangle$ for the reservoir, $\langle p-\psi, y\rangle$ for the turbine, and $\langle\psi, e\rangle$ for the river-when the shadow price $\psi$ is used to decentralise the operation within the plant (as is described in Section 3).

${ }^{24}$ That is, $\Pi(p ; \alpha k, \alpha e)=\alpha \Pi(p ; k, e)$ for every scalar $\alpha>0$. Note also that $\hat{Y}$ and $\hat{\Psi}$ are positively homogeneous, in $(k, e)$, of degrees 1 and 0 respectively; i.e., $\hat{Y}(p ; \alpha k, \alpha e)=\alpha \hat{Y}(p ; k, e)$ and $\hat{\Psi}(p ; \alpha k, \alpha e)=\hat{\Psi}(p ; k, e)$ for $\alpha>0$.

${ }^{25}$ When $\Pi$ is nondifferentiable, $\Pi(k, e)=r \cdot k+\langle\psi, e\rangle$ for every $(r, \psi) \in \widehat{\partial}_{k, e} \Pi$ (the superdifferential of $\Pi$ as a concave function of $(k, e))$.
} 
The marginal capacity values $\nabla_{k} \Pi_{\mathrm{SR}}^{\mathrm{H}}$ can be used to determine the optimum investment into a hydro plant on the basis of a given TOU electricity price $p$, a given river inflow $e$ and the supply costs of the two capital inputs, the reservoir and the turbine. Investment in reservoirs is well known to present a complicated problem which in practice often involves other considerations such as irrigation, flood control, navigation and leisure opportunities; and its scale can be large enough to affect an entire economy. What we outline here is investment planning for a purely hydroelectric scheme. The price-taking problem we formulate next is self-contained if the scheme is small enough not to change the existing price $p$. With a larger hydro scheme it can be used as part of a general equilibrium system that determines the new price as well. In any case, the turbine's unit cost, $r_{\mathrm{Tu}}$, can be reasonably regarded as constant, i.e., independent of the capacity $k_{\mathrm{Tu}}$. By contrast, the reservoir's marginal cost, $r_{\mathrm{St}}$, typically increases with $k_{\mathrm{St}}$ because the most suitable parts of the site are developed first. In formal terms, on a potential hydro site, a reservoir can be built at a cost which is a convex and increasing function, $G$, of its capacity $k_{\mathrm{St}} \in\left[0, \bar{k}_{\mathrm{St}}\right]$, with $G(0)=0$. Therefore, the investment problem is:

$$
\begin{aligned}
& \text { Given }\left(p, e, r_{\mathrm{Tu}}\right) \in \mathcal{C}[0, T] \times L^{\infty}[0, T] \times \mathbb{R}_{++} \text {and the function } G \\
& \text { maximize } \Pi_{\mathrm{SR}}^{\mathrm{H}}\left(p, k_{\mathrm{St}}, k_{\mathrm{Tu}}, e\right)-G\left(k_{\mathrm{St}}\right)-r_{\mathrm{Tu}} k_{\mathrm{Tu}} \text { over }\left(k_{\mathrm{St}}, k_{\mathrm{Tu}}\right) \in \mathbb{R}_{+}^{2}
\end{aligned}
$$

and the FOCs for an interior solution are:

$$
\begin{aligned}
& \frac{\partial \Pi_{\mathrm{SR}}^{\mathrm{H}}}{\partial k_{\mathrm{St}}}\left(k_{\mathrm{St}}, k_{\mathrm{Tu}}\right)=\frac{\mathrm{d} G}{\mathrm{~d} k_{\mathrm{St}}}\left(k_{\mathrm{St}}\right) \\
& \frac{\partial \Pi_{\mathrm{SR}}^{\mathrm{H}}}{\partial k_{\mathrm{Tu}}}\left(k_{\mathrm{St}}, k_{\mathrm{Tu}}\right)=r_{\mathrm{Tu}} .
\end{aligned}
$$

The system can be solved numerically (for $k_{\mathrm{St}}$ and $k_{\mathrm{Tu}}$ ) by, e.g., a quasi-Newton method: it requires no more than to calculate $\partial \Pi / \partial k_{\mathrm{St}}$ and $\partial \Pi / \partial k_{\mathrm{Tu}}$ at the successive approximations - and this can be done by applying Theorem 9 and solving the dual LP (21)-(27) or the unconstrained CP (33)-(35). A similar application to investment in pumped storage is presented in more detail in $[14]$.

Comment: For a multi-purpose reservoir, the upper and lower bounds on the water stock usually vary with time of year to ensure that the water level is high enough for navigation but low enough for holding any unexpected floodwater - so the constants $k_{\text {St }}$ and 0 in (17), etc., are replaced by some known functions of time, $\bar{s}(t)$ and $\underline{s}(t)$. Our results, including the uniqueness and continuity of the water value $\psi(t)$, extend to the case of slowly-varying reservoir constraints, i.e., it suffices to assume that both $\dot{\bar{s}}(t)$ and $\underline{\dot{s}}(t)$ always lie strictly between $-k_{\mathrm{Tu}}+e$ and $e$ (which are assumed to be negative and positive, respectively). Matters complicate if $\dot{\bar{s}}(t)$ or $\underline{\dot{s}}(t)$ are not confined to this range: the operating programme may even be infeasible, or $\psi$ may be discontinuous (and likely to be nonunique as well).

\section{The case of infeasible coasting}

With spillage assumed feasible as in (3), one can drop the condition that $e \leq k_{\mathrm{Tu}}$. But with $e \not \leq k_{\mathrm{Tu}}$, i.e., with coasting no longer feasible, an optimal water price $\psi$ need not be continuous or unique (despite the continuity of the electricity price $p$ ). 
For this extension, the primal problem (13)-(17) is modified by adding the spillage term, $\sigma \in L^{\infty}$, to the net outflow from the reservoir $f$, as in (1). The extra variable is constrained as in (3), i.e., $0 \leq \sigma \leq e$. However, there is no real need for an extra Lagrange multiplier for the constraint $\sigma \geq 0$ because such a multiplier would turn out to be identical to $\psi$ (at the dual optimum). The multiplier must be nonnegative; i.e., the constraint $\psi \geq 0$ must be adjoined to the dual (33)-(35). ${ }^{26}$ The multiplier for the constraint $\sigma \leq e$ turns out to be zero: the primal value is the same with or without this constraint. ${ }^{27}$ This means that free disposal of water is effectively unlimited, as in $[20,1.4 \mathrm{a}] .^{28}$ Last, an extra slackness condition, that $\psi=0$ a.e. on $\{t: \sigma(t)>0\}$, is adjoined to Part 2c of Proposition 3 .

In the extended framework, one can formally prove that an optimal storage policy involves no spillage if $k_{\mathrm{Tu}} \geq e$ and $p \in L_{++}^{1}$. This can be shown either by establishing that $\psi \gg 0$, or directly as follows. Suppose contrarily that $\sigma>0$ on a neighbourhood of some $t$. If $y(t)$ $<k_{\mathrm{Tu}}(t)$ then the output can be increased around $t$, so $(y, \sigma)$ is not optimal. If $y(t)=k_{\mathrm{Tu}}(t)$ then $\dot{s}(t)=(-y+e-\sigma)(t) \leq 0-\sigma(t)<0$, i.e., the stock is falling around $t$, and so there is room to store a unit being spilt, to release it at the nearest opportunity (which will come, since $\sigma \neq 0$ implies that $y(\tau)<e(\tau) \leq k_{\mathrm{Tu}}$ for some $\left.\tau\right)$. Again, this shows that $(y, \sigma)$ is not optimal. And although this argument treats $y, e$ and $\sigma$ as though they were continuous functions (rather than elements of $L^{\infty}$ ), it can be made rigorous by choosing $t$ to be a density point of the set $\left\{y<k_{\mathrm{Tu}}\right\}$ or $\left\{y=k_{\mathrm{Tu}}\right\}$, respectively. ${ }^{29}$

With $\operatorname{EssInf}(e)>0$ (but without assuming that $e \leq k_{\mathrm{Tu}}$ ), the modified primal and dual problems remain feasible, and the Kuhn-Tucker characterization of optimality continues to hold.$^{30}$ If the inflow exceeds the turbine's capacity only on a relatively short interval, spillage is still avoided. Consider an inflow increment $\left(k_{\mathrm{Tu}}-e\right)+\Delta e$ on an interval $[\underline{t}, \bar{t}]$ on which the reservoir is full in the original solution, the one that corresponds to an inflow $e<k_{\mathrm{Tu}}$. To make room for the excess inflow, an extra amount $\Delta E=\int_{t}^{\bar{t}} \Delta e(t) \mathrm{d} t$ of water is discharged immediately before $\underline{t}$, with the turbine operating at full capacity to sell the extra output at best prices, as close to $p(\underline{t})$ as possible. This solution is supported by the stock price $\psi$ that "freezes" when the discharge starts and stays constant until $\bar{t}$, when it jumps back to the original price trajectory (so $\psi$ is discontinuous at $\bar{t}$ ). As $\Delta E$ increases, so the discharge period preceding $[\underline{t}, \bar{t}]$ starts earlier. Here we assume that it does not merge with an earlier water collection period (during which $p<\psi$ ) before $\Delta E$ reaches $k_{\mathrm{St}} \cdot{ }^{31}$ In the borderline case of $\Delta E=k_{\mathrm{St}}$, the reservoir becomes empty at $\underline{t}$ and full again at $\bar{t}$. The no-spillage solution is still feasible, but only just; and the water price on $[\underline{t}, \bar{t}]$ is an arbitrary constant between 0 and $\psi(\underline{t}) .{ }^{32}$ In this case $\psi$ is nonunique (and discontinuous). If $\Delta E$ is further increased (keeping $\underline{t}$ and $\bar{t}$ fixed),

\footnotetext{
${ }^{26}$ This is superfluous when $e<k_{\text {Tu }}$ because in this case every solution, $\psi$, to (33)-(35) is nonnegative anyway.

${ }^{27}$ When $p \in L_{+}^{1}$, there is an optimum policy with $\sigma(t) \leq\left(e(t)-k_{\mathrm{Tu}}\right)^{+}<e(t)$.

${ }^{28}$ In reality, the spillage rate is constrained - quite apart from the considerations of flood control, etc.-by spillway capacity (unless this is exceeded by $e-y$ at a time when the reservoir is full and automatically overflows "from the top").

${ }^{29}$ For the concept of a density point, see, e.g., [8, (5.8)].

${ }^{30}$ Verification of Slater's Condition now requires a different choice of a feasible policy, viz., any $(y, \sigma)$ with $y+\sigma=e$ and $k_{\mathrm{Tu}}-\epsilon \geq y \geq \epsilon$ and $\sigma \geq \epsilon$, for some number $\epsilon>0$.

${ }^{31}$ If the two do merge, then the two constant values of $\psi$ become one value, which decreases as $\Delta E$ continues to increase (and the water collection period shrinks).

${ }^{32}$ This indeterminacy is noted in [20, p. 226: last paragraph].
} 
then a total of $\Delta E-k_{\mathrm{St}}$ must be spilt on $[\underline{t}, \bar{t}]$. This can be done in any way, but $\psi$ is unique (though it is discontinuous at $\underline{t}$ and $\bar{t}$ ), since $\psi=0$ on $[\underline{t}, \bar{t}]$.

\section{Conclusions}

This analysis shows how to operate a hydro plant to maximize its profit, how to value the plant's capacities and its river flow on this basis, and how to use these valuations in investment decisions. As well as being better suited to the more decentralized structure of today's utilities, short-run profit-maximization for an individual hydro plant turns out to be a much simpler problem to solve than that of cost minimization for a whole hydro-thermal system. When a hydro plant is operated to maximize profit, the hydro inputs (including the water inflow) have well defined marginal values, at least if the given TOU price for electricity is continuous over the cycle. The marginal capacity values and the TOU water value can be calculated by solving a linear programme (or an equivalent convex but unconstrained programme). These values can be used to determine the optimum levels of investment on a hydro site.

The "constant-head" model of the hydro technology has other interpretations as well: the analysis and its valuation method are applicable to other natural energy flows (e.g., geothermal or tidal), and also to water supply (when priced by TOU). An extension to the variable-head case (which requires convex control theory) is given in [17]. Extension to the case of stochastic river inflow is a subject for future work. This would especially enhance the model's application to water supply (as well as to the original hydro problem). 


\section{A Proofs}

Except for the shadow-price uniqueness result (Lemma 8), the proofs are mostly routine applications of duality for optimization in infinite-dimensional spaces, as expounded in, e.g., [24, Examples 4, 4', 4"] and [2, 3.3-3.7]. To put the primal constraints in the required operator form, define the integrals $I_{0}$ and $I_{T}: L^{\infty}[0, T] \rightarrow \mathcal{C}[0, T]$ by

$$
\left(I_{0} f\right)(t):=\int_{0}^{t} f(\tau) \mathrm{d} \tau \quad \text { and } \quad\left(I_{T} f\right)(t):=\int_{t}^{T} f(\tau) \mathrm{d} \tau .
$$

The reservoir constraints $(17)$ on $\left(y, s_{0}\right)$ can then be rewritten as

$$
0 \leq s_{0} 1_{[0, T]}-I_{0}(y-e) \leq k_{\mathrm{St}} .
$$

A formula for the adjoint operation $I_{0}^{*}: \mathcal{M}[0, T] \rightarrow L^{\infty *}[0, T]$ is needed. (As for the embedding $\mathbb{R} \ni s_{0} \mapsto s_{0} 1_{[0, T]} \in \mathcal{C}$, its adjoint is: $\mathcal{M} \ni \kappa \mapsto\langle\kappa, 1\rangle=\kappa[0, T]$.)

Lemma 10 The adjoints $I_{0}^{*}, I_{T}^{*}$ map $\mathcal{M}[0, T]$ into $\mathrm{BV}[0, T] \subset L^{1}[0, T]$; and they are given by

$$
\left(I_{0}^{*} \mu\right)(t)=\mu[t, T] \quad \text { and } \quad\left(I_{T}^{*} \mu\right)(t)=\mu[0, t] \quad \text { for a.e. } t
$$

for every $\mu \in \mathcal{M}[0, T]$. If $\mu[0, T]=0$, then $-I_{0}^{*} \mu=\mu[0, \cdot]=I_{T}^{*} \mu$.

Proof. This follows from Fubini's Theorem.

Proof of Theorem 1 (Fixed-input value minimization as the dual). Since (13)(17) is an LP, it would suffice to apply results such as those of [2, 3.3 and 3.6-3.7]. However, to facilitate extensions requiring nonlinear models, this proof is couched in $\mathrm{CP}$ terms. The dual to a concave maximization programme consists in minimizing, over the dual variables (the Lagrange multipliers for the primal), the supremum of the Lagrange function over the primal decision variables: see, e.g., [24, (4.6) and (5.13)]. The "cone model" of [24, Example 4'] is applicable, since (48) and (15)-(16) represent the inequality constraints of the primal programme (13)-(17) by means of the nonnegative cones $\left(\mathcal{C}_{+}\right.$and $\left.L_{+}^{\infty}\right)$ and convex constraint maps (which are actually linear).

The dual variables here are the $\kappa_{\mathrm{St}}, \nu_{\mathrm{St}} ; \kappa_{\mathrm{Tu}}, \nu_{\mathrm{Tu}}, \psi$ and $\lambda$ of (23); and these are paired with the parameter increments $\Delta k_{\mathrm{St}},-\Delta n_{\mathrm{St}}, \Delta k_{\mathrm{Tu}},-\Delta n_{\mathrm{Tu}}, \Delta e$ and $\Delta \zeta$ (as is discussed in Section 5). The primal variables are $\left(y, s_{0}\right) \in L^{\infty} \times \mathbb{R}$, and the Lagrange function is

$$
\mathcal{L}^{\mathrm{H}}\left(y, s_{0} ; \kappa, \nu, \psi, \lambda\right)= \begin{cases}\Pi_{\mathrm{Exc}}^{\mathrm{H}}\left(y, s_{0} ; \kappa, \nu, \lambda\right)+V^{\mathrm{H}}(\kappa, \psi) & \text { if }(\kappa, \nu) \geq 0 \text { and } \\ +\infty & \psi=\lambda-I_{0}^{*}\left(\kappa_{\mathrm{St}}-\nu_{\mathrm{St}}\right) \\ & \text { otherwise }\end{cases}
$$

where

$$
V^{\mathrm{H}}:=\left\langle\kappa_{\mathrm{St}}, k_{\mathrm{St}}\right\rangle_{\mathcal{M}, \mathcal{C}}+\left\langle\kappa_{\mathrm{Tu}}, k_{\mathrm{Tu}}\right\rangle_{L^{\infty *}, L^{\infty}}+\langle\psi, e\rangle_{L^{1}, L^{\infty}}
$$

and, with the notation

$$
\mu_{\mathrm{St}}:=\kappa_{\mathrm{St}}-\nu_{\mathrm{St}} \quad \text { and } \quad \mu_{\mathrm{Tu}}:=\kappa_{\mathrm{Tu}}-\nu_{\mathrm{Tu}}
$$


one has

$$
\begin{aligned}
\Pi_{\mathrm{Exc}}^{\mathrm{H}} & :=\left\langle p-\mu_{\mathrm{Tu}}-\lambda+I_{0}^{*} \mu_{\mathrm{St}}, y\right\rangle-\left\langle\mu_{\mathrm{St}}, s_{0}\right\rangle \\
& =\left\langle p-\mu_{\mathrm{Tu}}-\lambda+\mu_{\mathrm{St}}(\cdot, T], y\right\rangle-s_{0} \mu_{\mathrm{St}}[0, T]
\end{aligned}
$$

since $I_{0}^{*} \mu_{\mathrm{St}}=\mu_{\mathrm{St}}(\cdot, T]$ by Lemma 10 .

Formulae (50)-(53) are interpreted below; for details of their derivation, see [11, Appendix]. But first, to complete the calculation of the dual minimand when $(\kappa, \nu) \geq 0$ and

$$
\psi=\lambda-I_{0}^{*} \mu_{\mathrm{St}}
$$

(which are dual constraints, since the minimand is $+\infty$ otherwise), note that

$$
\sup _{y, s_{0}} \mathcal{L}=V+\sup _{y, s_{0}} \Pi_{\mathrm{Exc}}
$$

since $V$ is independent of $\left(y, s_{0}\right)$. By $(53), \Pi_{\mathrm{Exc}}$ is linear in these variables, so its supremum is either 0 or $+\infty$; and it is zero if and only if $\partial \Pi_{\mathrm{Exc}} / \partial s_{0}=0$ and $\nabla_{y} \Pi_{\mathrm{Exc}}=0$. These conditions are equivalent to the conjunction of $(25)$ and

$$
p=\lambda+\mu_{\mathrm{St}}[0, \cdot]+\mu_{\mathrm{Tu}} .
$$

In view of (25) and Lemma 10, (56) with (54) are the same as (26)-(27). So the dual programme is: given $(p ; k, e)$, minimize the $V(\kappa, \psi ; k, e)$ of $(51)$ over $(\kappa, \nu) \geq 0, \psi$ and $\lambda$ subject to (51)-(27).

Comments: (i) In (51)-(53), $V$ is the value of the available resources $(k, e)$, priced at $(\kappa, \psi)$.

(ii) For an entrepreneur buying all the inputs, $\Pi_{\text {Exc }}$ is the excess profit (a.k.a. pure profit) from an output $y$ and the use of an inflow $e$ and an initial stock $s_{0}$. To see this, recall from (50) that $0=\left\langle\lambda-\psi-I_{0}^{*} \mu_{\mathrm{St}}, e\right\rangle$, add this to (53) and use the identities $f(t)=y(t)-e(t)$ and $s(t)=s_{0}-I_{0} f(t)$ to obtain that

$$
\Pi_{\mathrm{Exc}}^{\mathrm{H}}=\langle p, y\rangle-\left\langle\kappa_{\mathrm{Tu}}-\nu_{\mathrm{Tu}}, y\right\rangle-\left\langle\kappa_{\mathrm{St}}-\nu_{\mathrm{St}}, s\right\rangle-\lambda\langle 1, f\rangle-\langle\psi, e\rangle .
$$

This sum is the total over the cycle of the revenue from sales to the market minus the cost of all the resources needed at each time $t$. The resources in question are: the time-varying minimum requirements for the turbine and reservoir capacities (priced at $\kappa$ ), the floors for generation and stock (priced at $\nu$ ), the required top-up (priced at $\lambda$ ), and the river inflow (priced at $\psi$ ). The last term in (57) can be rewritten as $\int_{0}^{T} \psi(t) e(t) \mathrm{d} t$, since $\psi \in L^{1}$ by $(27)$.

(iii) By adding and subtracting the value of internal sales (of the outflow $y$ from reservoir to turbine, priced at $\psi),(57)$ can be restated as

$$
\Pi_{\mathrm{Exc}}^{\mathrm{H}}=\langle p, y\rangle-\left\langle\mu_{\mathrm{Tu}}, y\right\rangle-\langle\psi, y\rangle+\langle\psi, y-e\rangle-\left\langle\mu_{\mathrm{St}}, s_{0}-I_{0}(y-e)\right\rangle-\langle\lambda, y-e\rangle .
$$

This gives $\Pi_{\mathrm{Exc}}$ as the sum of pure profits from the two parts of the plant: the first three terms add up to the excess profit from generation alone, whilst the other three terms add up to the excess profit from storage. The latter sum is equal to the appreciation of $s_{0}$ over the cycle because, with $\lambda-\psi=I_{0}^{*} \mu_{\mathrm{St}}$ and $f:=y-e$ as per (1) with $\sigma=0$,

$$
\langle\psi, f\rangle-\langle\lambda, f\rangle-\left\langle\mu_{\mathrm{St}}, s_{0}-I_{0}(f)\right\rangle=-\left\langle I_{0}^{*} \mu_{\mathrm{St}}, f\right\rangle-\left\langle\mu_{\mathrm{St}}, s_{0}-I_{0}(f)\right\rangle=-s_{0}\left\langle\mu_{\mathrm{St}}, 1\right\rangle .
$$


Proof of Remark 2. If this were false, then the minimand's value could be decreased by replacing $\left(\kappa_{\mathrm{St}}, \nu_{\mathrm{St}} ; \kappa_{\mathrm{Tu}}, \nu_{\mathrm{Tu}}\right)$ with $\left(\mu_{\mathrm{St}}^{+}, \mu_{\mathrm{St}}^{-} ; \mu_{\mathrm{Tu}}^{+}, \mu_{\mathrm{Tu}}^{-}\right)$given by $(52)$.

Proof of Proposition 3 (Dual solubility and optimality conditions). Like that of Theorem 1, this proof is put in CP terms. Consider first the dual problem with $L^{\infty *}$, instead of $L^{1}$, as the range for $\psi, \kappa_{\mathrm{Tu}}$ and $\nu_{\mathrm{Tu}}$ in (23). Since the nonnegative cones in the (primal) parameter spaces $\left(\mathcal{C}_{+}\right.$and $\left.L_{+}^{\infty}\right)$ have nonempty interiors (for the supremum norm), the framework of [24, Examples 4, 4', 4"] is applicable. To verify the Generalized Slater's Condition of $[24,(8.12)]$ for the primal constraints $(15)-(17)$, it suffices to take $y=e$ (so that $f=y-e$ $=0$ ), setting $s_{0}$ at any value strictly between 0 and $k_{\mathrm{St}}$. So the dual has a (proper) solution, and the primal and dual values are equal (and finite): see, e.g., [24, Theorems 18 (a) and 17 (a)].

To complete the proof of Part 1 , it remains to show that $\psi, \kappa_{\mathrm{Tu}}$ and $\nu_{\mathrm{Tu}}$ are in $L^{1}$. For $\psi$ this is obvious from (27). Next, from the Hewitt-Yosida decomposition of $(26)$ one has $\kappa_{\mathrm{Tu}}^{\mathrm{FA}}-\nu_{\mathrm{Tu}}^{\mathrm{FA}}$ $=p_{\mathrm{FA}}=0$, where $p_{\mathrm{FA}}$ means the purely finitely additive part of $p$ : see, e.g., [4, Appendix I: $(26)-(27)]$. Given (28), this means that $\kappa_{\mathrm{Tu}}^{\mathrm{FA}}=0=\nu_{\mathrm{Tu}}^{\mathrm{FA}}$, as required. (That $\nu_{\mathrm{Tu}}^{\mathrm{FA}}=0$ follows also from $p \geq 0$ alone: (28) and the Hewitt-Yosida decomposition of (26) give $\nu_{\mathrm{Tu}}^{\mathrm{FA}}=p_{\mathrm{FA}}^{-}=0$, as well as $\kappa_{\mathrm{Tu}}^{\mathrm{FA}}=p_{\mathrm{FA}}^{+}=p_{\mathrm{FA}}$.)

For Part 2, apply the Kuhn-Tucker saddle-point characterization of optima - given in, e.g., [24, Theorem 1 (e) and (f)] - to the primal (13)-(17) and its dual (21)-(27). This shows that $\left(y, s_{0}\right)$ and $(\kappa, \nu, \psi, \lambda)$ is a dual pair of solutions if and only if they maximize and minimize (respectively) the Lagrange function $\mathcal{L}$ given by (50). The minimum in question is characterized by: nonnegativity (24) and compatibility (27) of dual variables, primal feasibility (15)-(17) and complementary slackness, which translates here into Conditions $2 \mathrm{~b}$ and $2 \mathrm{c}$. As for the maximum in question, it is characterized by the conditions $\partial \Pi_{\mathrm{Exc}} / \partial s_{0}=0$ and $\nabla_{y} \Pi_{\mathrm{Exc}}=0$, i.e., by (25)(26).

Comment: Existence of a dual optimum in the norm-dual spaces $\left(\kappa_{\mathrm{St}}\right.$ and $\nu_{\mathrm{St}}$ in $\mathcal{M}=\mathcal{C}^{*}$, and $\kappa_{\mathrm{Tu}}, \nu_{\mathrm{Tu}}$ and $\psi$ in $L^{\infty *}$ ) comes automatically from (20), which ensures that the Generalized Slater's Condition of $[24,(8.12)]$ holds with the norm topologies of the primal parameter spaces $L^{\infty}$ and $\mathcal{C}$. The density representation (of the dual variables other than $\kappa_{\mathrm{St}}$ and $\nu_{\mathrm{St}}$ ) comes from the problem's structure and the assumptions on $p$ : by the constraint $(27), \psi \in \mathrm{BV} \subset L^{1}$; with $p \geq 0$, every optimal $\nu_{\mathrm{Tu}}$ is in $L^{1}$; and if $p \in L^{1}$ then every optimal $\kappa_{\mathrm{Tu}}$ is also in $L^{1}$.

Proof of Proposition 4 (Shadow pricing of water as the dual). This is a reformulation of Theorem 1: substitute the $\psi$ given by (27) into (26), and note that, given any $\psi$ (and $p$ ), the best choice for $\kappa_{\mathrm{St}}$ and $\kappa_{\mathrm{Tu}}$ is as in (31)-(32), because $k_{\mathrm{St}}>0$ and $k_{\mathrm{Tu}}>0$. This reduces the dual programme $(21)-(27)$ to minimization of

$$
k_{\mathrm{St}} \int_{[0, T]}(\mathrm{d} \psi)^{+}+k_{\mathrm{Tu}} \int_{0}^{T} \kappa_{\mathrm{Tu}}(\mathrm{t}) \mathrm{d} t+\int_{0}^{T} \psi(t) e(t) \mathrm{d} t
$$

over $\psi \in \mathrm{BV}[0-, T+]$, subject to $\psi(0-)=\psi(T+)$ lying between $\psi(0+)$ and $\psi(T-)$. Hence the first of the integrals equals the sum of $(\psi(0+)-\psi(T-))^{+}$and $\int_{(0, T)}(\mathrm{d} \psi)^{+}$; and this sum is $\operatorname{Var}_{\mathbf{c}}^{+}(\psi)$.

Proof of Proposition 5 (Primal solubility). With $p \in L^{1}$, the maximand of (14) is continuous for the weak* topology $\mathrm{w}\left(L^{\infty}, L^{1}\right)$. The feasible set is bounded: in $y$ by $(15)$, and 
in $s_{0}$ by (17) with, e.g., $t=0$. So, being also weakly* closed, the feasible set is compact by the Banach-Alaoglu Theorem. And it is nonempty, since the point $\left(y, s_{0}\right)=(e, 0)$ is feasible by assumption. So an optimum exists by Weierstrass's Extreme Value Theorem.

At this stage, it is useful to introduce a notation for the sets of those times when the reservoir is empty or full or neither, given a hydro output $y$ meeting the balance constraint $\int_{0}^{T} f(t) \mathrm{d} t$ $=0$. These sets (which have already appeared in Condition $2 \mathrm{~b}$ of Proposition 3 ) are:

$$
\begin{aligned}
E(f) & :=\{t \in[0, T]: s(t)=0\} \\
F\left(f, k_{\mathrm{St}}\right) & :=\left\{t \in[0, T]: s(t)=k_{\mathrm{St}}\right\} \\
B\left(f, k_{\mathrm{St}}\right) & :=[0, T] \backslash(E \cup F)=\left\{t: 0<s(t)<k_{\mathrm{St}}\right\}
\end{aligned}
$$

where $s(t)$ is given by (18)-(19) in terms of $f:=y-e$, and $k_{\mathrm{St}} \geq \operatorname{Max}(s)$. Since $s(0)=s(T)$, 0 and $T$ are either both in $B$, or both in $E$, or both in $F$. From (18), $E \neq \emptyset$. Unless there is spare reservoir capacity, $F \neq \emptyset$ also; and then all three sets are nonempty. Their connected components are subintervals of $[0, T]$; and, being open, $B$ is the union of a countable (finite or denumerable) sequence of intervals. Those not containing 0 or $T$ are denoted by

$$
A_{m}=\left(\underline{t}_{m}, \bar{t}_{m}\right) \neq \emptyset
$$

for $m=1, \ldots, M \leq \infty$, where $0 \leq \underline{t}_{m}<\bar{t}_{m} \leq T$. If $\{0, T\} \subseteq B$, then $B$ additionally contains two subintervals whose union is

$$
A_{0}=\left(\underline{t}_{0}, T\right] \cup\left[0, \bar{t}_{0}\right)
$$

for some $0<\bar{t}_{0}<\underline{t}_{0}<T$. When $0, T \notin B$, we set for completeness $\underline{t}_{0}=T$ and $\bar{t}_{0}=0$, so that $A_{0}=\emptyset$ in this case. In either case $B=\bigcup_{m \geq 0} A_{m}$.

All these sets may be thought of as subsets of the circle that results from "gluing" 0 and $T$ into a single point $T 0$. Then $\left(A_{m}\right)_{m \geq 0}$ are the component arcs of $B$ (a.k.a. $B$-arcs); $A_{0}$ is that arc which contains $T 0$ (if $T 0 \in B$ ); and $\underline{t}_{m}$ and $\bar{t}_{m}$ are the beginning and the end of arc $A_{m}$ (w.r.t. the "clockwise" orientation).

The formula for the output $y$, in terms of any $\psi \in \hat{\Psi}$, is proved next. On $\{t: p \neq \psi\}$, the optimal $y$ equals unambiguously $k_{\mathrm{Tu}}$ or 0 . Uniqueness of $y$ on $\{p=\psi\}$ comes from the no-plateau assumption (36) on $p$ : this ensures that $\{p=\psi\} \subseteq E \cup F$, up to a null set. And at each $t \in E \cup F$ one has $f(t)=-\dot{s}(t)=0$ (and hence $y(t)=e(t)$ ), since $s$ has an extremum at $t$.

Remark 11 If $s:[0, T] \rightarrow[0,1]$ is absolutely continuous, then $\dot{s}=0$ almost everywhere on the set $E:=\{t \in[0, T]: s(t)=0\}$.

Proof of Proposition 6 (Hydro output with plateau-less price). Take any $y \in \hat{Y}$ (not yet known to be unique) and any $\psi \in \hat{\Psi}$ (which may be nonunique, unless $p \in \mathcal{C}$ ). The first and the third lines of (37) follow from Part 2c of Proposition 3 with (26)-(27). It remains to show that $y=e$ a.e. on $S:=\{t: p=\psi\}$. For each $m$, one has $\psi=$ const. on $A_{m}\left(f, k_{\mathrm{St}}\right)$ by Part $2 \mathrm{~b}$ of Proposition 3. Therefore meas $\left(S \cap A_{m}\right)=0$ by (36), and hence meas $\left(S \cap B\left(f, k_{\mathrm{St}}\right)\right)$ $=0$ by countable additivity. This means that $S$ is, up to a null set, contained in the set $F\left(f, k_{\mathrm{St}}\right) \cup E(f)$, on which $y-e=-\dot{s}=0$ a.e. (by Remark 11). This completes the proof of 
(37). It follows that $\hat{Y}$ is a singleton, even when $\hat{\Psi}$ is not. (Given any $\psi \in \hat{\Psi}$, any $y^{\prime}$ and $y^{\prime \prime}$ from $\hat{Y}$ satisfy (37) and are therefore equal.)

Proof of Corollary 7 (Dual calculation of SR profit). Formula (38) follows from Propositions 3 and 4 . To derive it term-by-term, use the optimality conditions (complementary slackness and feasibility) to expand $\langle p, y\rangle$ :

$$
\begin{aligned}
\Pi & :=\int_{0}^{T} p y \mathrm{~d} t=\int_{0}^{T}(p-\psi) y \mathrm{~d} t+\int_{0}^{T} \psi e \mathrm{~d} t+\int_{0}^{T} \psi(y-e) \mathrm{d} t \\
& =k_{\mathrm{Tu}} \int_{0}^{T}(p-\psi)^{+} \mathrm{d} t+\int_{0}^{T} \psi e \mathrm{~d} t-\int_{0}^{T} \psi \frac{\mathrm{d} s}{\mathrm{~d} t} \mathrm{~d} t
\end{aligned}
$$

integrating the last term by parts to obtain

$$
\begin{aligned}
-\int_{0}^{T} \psi \mathrm{d} s & =-[\psi s]_{t=0-}^{t=T+}+\int_{[0, T]} s \mathrm{~d} \psi=s(0)(\psi(0-)-\psi(T+))+k_{\mathrm{St}} \int_{[0, T]}(\mathrm{d} \psi)^{+} \\
& =0+k_{\mathrm{St}} \operatorname{Var}_{\mathrm{c}}^{+}(\psi)
\end{aligned}
$$

as required.

Before a detailed proof of Lemma 8, it is worth presenting the main ideas. The key principle is that equipment can earn a rent only at a time of full capacity utilization. In the present context this means that $p$ can exceed $\psi$ only when the turbine is working at full power (i.e., when $y(t)=k_{\mathrm{Tu}}$ ). Similarly $\psi$ can exceed $p$ only when the turbine is off (i.e., when $y(t)$ $=0)$. Therefore $\psi(t)$ equals $p(t)$ when the reservoir is either full or empty (since $s(t)=0$ or $s(t)=k_{\mathrm{St}}$ implies that $y(t)=-\dot{s}(t)+e(t)=e(t)$, which lies strictly between 0 and $k_{\mathrm{Tu}}$ by assumption). By the same principle, $\psi$ can be rising or falling only when the reservoir is full or empty (respectively); so $\psi$ stays constant on each open interval $(\underline{t}, \bar{t})$ during which the reservoir constraints are inactive (i.e., $0<s(t)<k_{\mathrm{St}}$ ). Together, these conditions determine the function $\psi$ almost completely - except for the possibility of jumps or drops of $\psi$ that may occur at endpoints of a (closed) interval on which the reservoir is either full throughout or empty throughout. ${ }^{33}$ Suppose, for example, that $\underline{t}$ is the end of an interval on which the reservoir is full. At that instant, $\psi$ can jump but not drop; and the same is true of $p-\psi$ (since $p=\psi$ just before $\underline{t}$, and $p \geq \psi$ just after $\underline{t}$ ). So neither term, $\psi$ or $p-\psi$, can jump at $\underline{t}$ if their sum $(p)$ is continuous. This determines the constant value of $\psi$ on $(\underline{t}, \bar{t})$ as $p(\underline{t})$; so $\psi$ is unique.

Proof of Lemma 8 (Water price uniqueness and continuity). Fix any primal solution $y \in \hat{Y}$, which exists by Proposition 3 (though it may be nonunique). To show that there is just one dual solution, we shall express every dual solution $\psi \in \hat{\Psi}$ by the same formula in terms of $y .{ }^{34}$

In the case of $F\left(y, k_{\mathrm{St}}\right) \neq \emptyset$, which we deal with first, we shall use the Kuhn-Tucker Conditions to show that any $\psi \in \hat{\Psi}\left(p, k_{\mathrm{H}}, e\right)$ can be given, in terms of $y$, as

$$
\psi(t)=p(t) \quad \text { for every } t \in(E \cup F)\left(f, k_{\mathrm{St}}\right) \backslash\{0, T\}
$$

\footnotetext{
${ }^{33}$ To simplify, we assume here that the times when the reservoir is full form a set $F$ that consists of a finite number of intervals (which may be single instants). Although $F$ can be more complex, this is only a technicality (dealt with in the Proof of Lemma 8).

${ }^{34}$ The basis for this strategy (used also in proving Proposition 6) is that every dual solution supports every primal solution; i.e., the set of saddle-points for a dual pair of convex programmes is the Cartesian product (of the primal and dual solution sets): see Proposition 3.
} 
whereas on the $m$-th component $A_{m}$ of $B\left(f, k_{\mathrm{St}}\right)$, whose endpoints are $\underline{t}_{m}$ and $\bar{t}_{m}$, it is the constant

$$
\psi(t)=\left\{\begin{array}{ll}
p\left(\underline{t}_{m}\right) & \text { if } \underline{t}_{m} \neq 0 \\
p\left(\bar{t}_{m}\right) & \text { if } \bar{t}_{m} \neq T
\end{array} \quad \text { for every } t \in A_{m}\left(f, k_{\mathrm{St}}\right)\right.
$$

for each $m \geq 0$. Since both $E$ and $F$ are nonempty, $A_{m} \neq(0, T)$, so at least one line of (62) applies; and when both do, they are consistent. So (61)-(62) fully determine $\psi$ on $(0, T)$, and hence on $[0, T]$ because $\psi(0)$ and $\psi(T)$ are defined by continuity.

To use the optimality conditions as stated in Proposition 3 -i.e., in terms of $(\kappa, \nu, \psi, \lambda)$ rather than $\psi$ alone - recall from Section 7 that if a $\psi \in \mathrm{BV}(0, T)$ solves $(33)-(35)$, then (21)(27) is solved by: the same $\psi,\left(\kappa_{\mathrm{Tu}}, \nu_{\mathrm{Tu}}\right)=\left((p-\psi)^{+},(p-\psi)^{-}\right)$, any $\lambda$ between $\psi(0+)$ and $\psi(T-)$ and $\left(\kappa_{\mathrm{St}}, \nu_{\mathrm{St}}\right)=\left(\mu_{\mathrm{St}}^{+}, \mu_{\mathrm{St}}^{-}\right)$, where $\mu_{\mathrm{St}}=\mathrm{d} \psi$ on $(0, T)$ with $\mu\{0\}=\psi(0+)-\lambda$ and $\mu\{T\}$ $=\lambda-\psi(T-)$.

By (26)-(27),

$$
p=\psi+\kappa_{\mathrm{Tu}}-\nu_{\mathrm{Tu}}=\lambda+\left(\kappa_{\mathrm{St}}-\nu_{\mathrm{St}}\right)[0, \cdot]+\kappa_{\mathrm{Tu}}-\nu_{\mathrm{Tu}} \quad \text { a.e. }
$$

It suffices to show that, at every point of $(E \cup F) \backslash\{0, T\}, \psi$ is continuous and equal to $p$ : then (62) follows, since $\psi$ is constant on each $B$-component $A_{m}$, and since $A_{m} \neq(0, T)$.

A discontinuity of $\psi$ could only be a jump at a time when the reservoir is full, or a drop when it is empty. If $t \in F$ say, then, being full at $t$, the reservoir cannot be being emptied just before $t{ }^{35}$ That is, just before $t$ the outflow $y$ cannot exceed the inflow $e$, which, by assumption, is smaller than $k_{\mathrm{Tu}}$. A fortiori, the capacity charge $\kappa_{\mathrm{Tu}}$ must be zero just before $t$. Similarly, just after a $t \in F$ the reservoir cannot be being filled, i.e., $y$ cannot be less than $e$, which is positive by assumption; and so $\nu_{\mathrm{Tu}}$ must be zero just after $t$. So $p-\psi=\kappa_{\mathrm{Tu}}-\nu_{\mathrm{Tu}}$ is nonpositive just before $t$ and nonnegative just after $t$, and hence $p-\psi$ cannot drop at a $t \in F$. This means that any discontinuous changes in $\psi$ and $p-\psi$ are of the same sign and cannot cancel each other out. So $\psi$ (and $p-\psi$ ) must be continuous if $p$ is. And it follows (from the signs of $p-\psi$ before and after $t$ ) that $p(t)=\psi(t)$. The "upside down" version of this reasoning applies to $t \in E$.

Since $\kappa_{\mathrm{Tu}}$ and $\nu_{\mathrm{Tu}}$ are equivalence classes, this argument is formalized by using the essential limit concept-for which see, e.g., [6, IV.36-IV.37] or [25, II.9: p. 90]. It is also convenient to say that an inequality between functions holds somewhere on $A \subseteq[0, T]$ to mean that it holds on an $A^{\prime} \subseteq A$ with meas $A^{\prime}>0$ (i.e., it is not the case that the reverse inequality holds a.e. on A).

Recall from Section 4 that $y$ with the $\underline{s}_{0, y}$ of (18) solve (13)-(17). Consider first a $t \in$ $F \backslash\{0, T\}$. For every $\Delta t>0$, it cannot be that $f>0$ a.e. on $(t-\Delta t, t)$; i.e., somewhere on $(t-\Delta t, t)$ one has $y \leq e<k_{\mathrm{Tu}}$. Therefore $\kappa_{\mathrm{Tu}}=0$ somewhere on $(t-\Delta t, t)$, by Part 2c of Proposition 3; and, as $\Delta t \rightarrow 0$, this shows that the lower left essential limit of $\kappa_{\mathrm{Tu}}$ at $t$ is zero. Similarly, somewhere on $(t, t+\Delta t)$ one has $f \geq 0$, i.e., $y \geq e>0$. Therefore $\nu_{\mathrm{Tu}}=0$ somewhere on $(t, t+\Delta t)$. This means that the lower right essential limit of $\nu_{\text {Tu }}$ at $t$ is zero; i.e.,

$$
\operatorname{ess} \liminf _{\tau \searrow t} \nu_{\mathrm{Tu}}(\tau)=0=\operatorname{ess} \liminf _{\tau / t} \kappa_{\mathrm{Tu}}(\tau) \quad \text { for } t \in F \backslash\{0, T\} .
$$

\footnotetext{
${ }^{35}$ This, by the way, is where the constancy of $k_{\text {St }}$ over time is used.
} 
Given (63) as well as continuity of $p$ and nonnegativity of $\kappa_{\mathrm{Tu}}$ and $\nu_{\mathrm{Tu}}$, it follows from (64) that $^{36}$

$$
\begin{aligned}
p(t)-\psi(t-) & =\operatorname{ess} \lim _{\tau \nearrow t}\left(\kappa_{\mathrm{Tu}}-\nu_{\mathrm{Tu}}\right)(\tau) \\
& =\operatorname{ess} \liminf _{\tau \nearrow t} \kappa_{\mathrm{Tu}}(\tau)-\operatorname{ess} \liminf _{\tau \nearrow t} \nu_{\mathrm{Tu}}(\tau) \leq 0 \\
& \leq \operatorname{ess} \liminf _{\tau \searrow t} \kappa_{\mathrm{Tu}}(\tau)-\operatorname{ess} \liminf _{\tau \searrow t} \nu_{\mathrm{Tu}}(\tau)=\operatorname{ess} \lim _{\tau \searrow t}\left(\kappa_{\mathrm{Tu}}-\nu_{\mathrm{Tu}}\right)(\tau) \\
& =p(t)-\psi(t+) .
\end{aligned}
$$

Therefore $\psi(t-) \geq \psi(t+)$ from a comparison of the first and the last sums. But also, since $t \in F$,

$$
\psi(t-) \leq \psi(t+)
$$

by Part $2 \mathrm{~b}$ of Proposition 3; so all three inequalities (65), (66) and (67) must actually hold as equalities. This shows that $\psi(t-)=\psi(t+)=p(t)$, i.e., the two-sided limit of $\psi$ at $t$ exists and equals $p(t)$. (Since it exists, it also equals $\psi(t)$ because $\psi(t)$ always lies between $\psi(t-$ ) and $\psi(t+)$.) The same can be shown for $t \in E$ (by the "upside down" version of the proof for $t \in F)$; so

$$
\psi(t)=\lim _{\tau \rightarrow t} \psi(\tau)=p(t) \quad \text { for } t \in(E \cup F) \backslash\{0, T\} \neq \emptyset .
$$

Nonemptiness of this set follows from the assumption that $F \neq \emptyset$, since $E \neq \emptyset$ always, by (18).

By Part $2 \mathrm{~b}$ of Proposition $3, \psi$ is constant on each $A_{m}$. This and (68) show that $\psi \in \mathcal{C}(0, T)$. (Equivalently $\psi \in \mathcal{C}[0, T]$, since $\psi(0):=\psi(0+)$ and $\psi(T):=\psi(T-)$.)

It remains to show that the proven properties of $\psi$ imply (62). Since $E \cup F \nsubseteq\{0, T\}$, the set $B$ consists of two or more nonempty components $A_{m}$. Each of these has at least one endpoint that is neither 0 nor $T$; i.e., $\underline{t}_{m} \neq 0$ or $\bar{t}_{m} \neq T\left(\underline{t}_{m} \neq T\right.$ and $\bar{t}_{m} \neq 0$ always). Say it is $\underline{t}_{m}$; then $\underline{t}_{m} \in(E \cup F) \backslash\{0, T\}$, since $\underline{t}_{m} \notin A_{m}\left(A_{m}\right.$ is an open arc). So, by (68) and the constancy of $\psi$ on $A_{m}$,

$$
p\left(\underline{t}_{m}\right)=\psi\left(\underline{t}_{m}\right)=\psi(t) \quad \text { for every } t \in A_{m} .
$$

If $T \neq \bar{t}_{m}$, then (69) holds with $\bar{t}_{m}$ in place of $\underline{t}_{m}$, by the same argument. This also shows that $p\left(\underline{t}_{m}\right)=p\left(\bar{t}_{m}\right)$ if both $\underline{t}_{m} \neq 0$ and $\bar{t}_{m} \neq T$. (All this applies to $m=0$ as well, if $A_{0} \neq \emptyset$. In this case $\psi$ is additionally constant on $A_{0} \supset\{0, T\}$; so $\psi(0)=\psi(T)$ even if $p(0) \neq p(T)$.) This completes the proof of $(61)-(62)$ when $F \neq \emptyset$.

If $p(0)=p(T)$, then $\psi(0)=\psi(T)$ follows by virtually the same argument as that proving (68), with 0 and $T$ thought of as a single point of the circle.

Finally, consider the case of $F\left(f, k_{\mathrm{St}}\right)=\emptyset$, which is trivial in that the reservoir is never used to capacity, and it earns no rent. Formally, $\kappa_{\mathrm{St}}=\nu_{\mathrm{St}}=0$ by Part $2 \mathrm{~b}$ of Proposition 3 and (25); so $\psi$ is a constant. Its uniqueness is readily shown: $\psi$ minimizes $(34)$ over $\mathrm{BV}(0, T)$, so, a fortiori, it minimizes (34) over $\mathbb{R}$. Since for $\psi \in \mathbb{R}$ the sum (34) simplifies to

$$
k_{\mathrm{Tu}} \int_{0}^{T}(p(t)-\psi)^{+} \mathrm{d} t+\psi \int_{0}^{T} e(t) \mathrm{d} t
$$

\footnotetext{
${ }^{36}$ This argument uses also the fact that $\lim \inf (A-B) \leq \lim \inf A-\lim \inf B \leq \lim \sup (A-B)$ whenever the middle term is well defined. It equals $\lim (A-B)$ if the latter exists, as here (although the inequalities suffice). The same holds with $\lim \sup A-\lim \sup B$ as the middle term.
} 
the minimum in question is characterized by the FOC

$$
\text { meas }\{t: p(t)>\psi\} \leq \frac{1}{k_{\mathrm{Tu}}} \int_{0}^{T} e(t) \mathrm{d} t \leq \operatorname{meas}\{t: p(t) \geq \psi\}
$$

which means that $\psi$ is an upper quantile of order $\left(1 / T k_{\mathrm{Tu}}\right) \int_{0}^{T} e(t) \mathrm{d} t$ for the distribution of $p$ with respect to meas $/ T .{ }^{37}$ And the quantile is unique if $p \in \mathcal{C}[0, T]$, since the cumulative distribution function of $p$ is then strictly increasing on the interval (Min $(p), \operatorname{Max}(p)$ ).

Comment: Although (64) suffices for the argument, both inf signs can be deleted, i.e., (64) can be strengthened to: $\kappa_{\mathrm{Tu}}(t-)=0=\nu_{\mathrm{Tu}}(t+)$ with $\nu_{\mathrm{Tu}}(t-) \geq 0$ and $\kappa_{\mathrm{Tu}}(t+) \geq 0$, for $t \in F \backslash\{0, T\}$, whenever $p(t \pm)$ exist. ${ }^{38}$ This is because, by (28) and the continuity of $\kappa \mapsto \kappa^{ \pm} \in \mathbb{R}_{+}$, the four limits exist and are equal to $\left(\kappa_{\mathrm{Tu}}-\nu_{\mathrm{Tu}}\right)^{ \pm}(t \pm)=(p-\psi)^{ \pm}(t \pm)$. All four limits are zero if $p$ is continuous at $t$.

Given Lemma 8, Theorem 9 is a routine case of the marginal interpretation of the dual solution. Before a formal proof, it is worth retracing in the present context the familiar argument which establishes the derivative property of the value function when differentiability is taken for granted. With the dual minimand (34) denoted by $V\left(k_{\mathrm{H}}, e, \psi\right)$, the r.h.s.'s of $(40)-(42)$ are obviously the partial derivatives of $V$ in $\left(k_{\mathrm{H}}, e\right)$ evaluated at the dual optimum $\hat{\psi}\left(k_{\mathrm{H}}, e\right)$. And the total derivatives, in $\left(k_{\mathrm{H}}, e\right)$, of the dual value $V\left(k_{\mathrm{H}}, e, \hat{\psi}\left(k_{\mathrm{H}}, e\right)\right)$ are equal to the corresponding partial derivatives, since the partial derivative of $V$ in $\psi$ vanishes by the FOC for the optimality of $\hat{\psi}$. To complete the calculation, note that the dual value equals the primal value $\Pi_{\mathrm{SR}}^{\mathrm{H}} \cdot{ }^{39}$ This is, indeed, the substance of the first step in the Proof of Theorem 9, except that a standard convex duality result is used instead of the above derivation "from first principles". This is necessary because a rigorous application of the chain rule would run into difficulties, since it would require the differentiability of $\hat{\psi}$ in $\left(k_{\mathrm{H}}, e\right)$, and of $V$ in $\psi$. This would make their composition $\Pi\left(k_{\mathrm{H}}, e\right)=V\left(k_{\mathrm{H}}, e, \hat{\psi}\left(k_{\mathrm{H}}, e\right)\right)$ differentiable, but neither this nor even the uniqueness of an optimal $\psi$ (i.e., the existence of $\hat{\psi}$ ) may be presupposed. Rather, these properties must be derived - by using price continuity, since they are known to fail in general if $p \notin \mathcal{C}$ (see [11] for an example). This gap is filled by Lemma 8 .

Proof of Theorem 9 (Efficiency rents of a hydro plant). The first, routine, step is to identify the dual variables as marginal values of the primal parameters, with the marginal values formalized as supergradients (of the primal value, a concave function of the parameters): see, e.g., [24, Theorem 16: (b) and (a), with Theorem 15: (e) and (f)] or [18, 7.3: Theorem 1']. This is applied in such a way as to give the marginal interpretation to the optimal $\kappa$ and $\nu$ themselves, rather than only to their totals over the cycle, although the formulae to be proved are for the total values. Therefore the short-run profit is considered as a function, $\widetilde{\Pi}_{\mathrm{SR}}^{\mathrm{H}}$, of all the quantity parameters

$$
\left(\Delta k_{\mathrm{St}}, \Delta n_{\mathrm{St}} ; \Delta k_{\mathrm{Tu}}, \Delta n_{\mathrm{Tu}} ; \Delta e, \Delta \zeta\right) \in \mathcal{C} \times \mathcal{C} \times L^{\infty} \times L^{\infty} \times L^{\infty} \times \mathbb{R}
$$

\footnotetext{
${ }^{37}$ Note that $0<\int_{0}^{T} e(t) \mathrm{d} t<T k_{\mathrm{Tu}}$ by $(20)$.

${ }^{38}$ The abbreviations $\kappa(t \pm)$ for the essential (one-sided) limits should not be mistaken for the ordinary limits of a particular variant of $\kappa$, in as much as the ordinary limits may be nonexistent.

${ }^{39}$ Conversely, the equality of short-run profit to the fixed-input value can be rederived from (40)-(42) by applying Euler's Theorem to $\Pi$ as a jointly homogeneous function of $(k, e)$.
} 
discussed in Section 5. It is an extension of the optimal value of the programme (13)-(17), i.e.,

$$
\Pi_{\mathrm{SR}}^{\mathrm{H}}\left(p ; k_{\mathrm{St}}, k_{\mathrm{Tu}}, e\right)=\widetilde{\Pi}_{\mathrm{SR}}^{\mathrm{H}}\left(p ; k_{\mathrm{St}}, 0 ; k_{\mathrm{Tu}}, 0 ; e, 0\right) \quad \text { for }\left(k_{\mathrm{St}}, k_{\mathrm{Tu}}\right) \in \mathbb{R}^{2}
$$

where the scalars are identified with constant functions on $[0, T]$. In this setting, the result giving the marginal values of the primal parameters is

$$
\begin{array}{r}
\widehat{\partial}_{k_{\mathrm{St}}, n_{\mathrm{St}}, k_{\mathrm{Tu}}, n_{\mathrm{Tu}}, e, \zeta} \widetilde{\Pi}_{\mathrm{SR}}^{\mathrm{H}}=\left\{\left(\kappa_{\mathrm{St}},-\nu_{\mathrm{St}}, \kappa_{\mathrm{Tu}},-\nu_{\mathrm{Tu}}, \psi, \lambda\right):(\kappa, \nu, \psi, \lambda)\right. \\
\text { meet Conditions } 2 \mathrm{a}, 2 \mathrm{~b} \text { and } 2 \mathrm{c} \text { of Proposition } 3\} .
\end{array}
$$

For differentiation of $\Pi_{\mathrm{SR}}^{\mathrm{H}}$, with respect to the constant capacities and the cyclically varying inflow, it follows from (71) that

$$
\begin{aligned}
\widehat{\partial}_{k_{\mathrm{St}}, k_{\mathrm{Tu}}, e} \Pi_{\mathrm{SR}}^{\mathrm{H}} & =\left\{\left(\int_{[0, T]} \kappa_{\mathrm{St}}(\mathrm{d} t), \int_{0}^{T} \kappa_{\mathrm{Tu}}(t) \mathrm{d} t, \psi\right): \exists \nu \exists \lambda(\kappa,-\nu, \psi, \lambda) \in \widehat{\partial}_{k_{\mathrm{H}}, n_{\mathrm{H}}, e, \zeta} \widetilde{\Pi}_{\mathrm{SR}}^{\mathrm{H}}\right\} \\
& =\left\{\left(\operatorname{Var}_{\mathrm{c}}^{+}(\psi), \int_{0}^{T}(p-\psi)^{+} \mathrm{d} t, \psi\right): \psi \in \hat{\Psi}\left(p ; k_{\mathrm{St}}, k_{\mathrm{Tu}}, e\right)\right\}
\end{aligned}
$$

by using (32) and substituting $\kappa_{\mathrm{St}}=(\mathrm{d} \psi)^{+}$. When $p \in \mathcal{C}$, the set $\hat{\Psi}$ in (72) is actually a singleton by Lemma 8 , and hence so is $\widehat{\partial}_{k_{\mathrm{H}}, e} \Pi_{\mathrm{SR}}^{\mathrm{H}}\left(p ; k_{\mathrm{H}}, e\right)$. 


\section{References}

[1] Anderson, D. (1976): "Models for determining least-cost investments in electricity supply", Bell Journal of Economics, 7, 267-299.

[2] Anderson, E. J., and P. Nash (1987): Linear programming in infinite-dimensional spaces. New York-Chichester-Brisbane-Toronto-Singapore: Wiley. Applied nonlinear analysis. New York: Wiley.

[3] Bauer, W., H. Gfrerer, and H. Wacker (1984): "Optimization strategies for hydro energy storage plants", Zeitschrift für Operations Research, Series B, 28, 103-131.

[4] Bewley, T. (1972): "Existence of equilibria in economies with infinitely many commodities", Journal of Economic Theory, 4, 514-540.

[5] Boiteux, M. (1964): "Peak-load pricing", in Marginal cost pricing in practice (Chapter 4), ed. by J. R. Nelson. Engelwood Cliffs, NJ: Prentice Hall. (A translation of "La tarification des demandes en pointe: application de la théorie de la vente au cout marginal", Revue Général de l'Electricité, 58 (1949), 321-340.)

[6] Dellacherie, C., and P. A. Meyer (1978): Probabilities and potential. Amsterdam-New York-Oxford: North-Holland.

[7] El-Hawary, M. E., and G. S. Christensen (1979): Optimal economic operation of electric power systems. New York-San Francisco-London: Academic Press.

[8] Foran, J. (1991): Fundamentals of real analysis. New York-Basel-Hong Kong: Dekker.

[9] Gfrerer, H. (1984): "Optimization of hydro energy storage plants by variational methods", Zeitschrift für Operations Research, Series B, 28, 87-101.

[10] Horsley, A., and A. J. Wrobel (1988): "Subdifferentials of convex symmetric functions: An application of the inequality of Hardy, Littlewood and Pólya", Journal of Mathematical Analysis and Applications, 135, 462-475.

[11] Horsley, A., and A. J. Wrobel (1999): "Efficiency rents of storage plants in peak-load pricing, II: hydroelectricity", STICERD Discussion Paper TE/99/372, LSE.

[12] Horsley, A., and A. J. Wrobel (1999): The Current State of Economic Science (Volume 1, pp. 453-480). Spellbound Publications: Rohtak.

[13] Horsley, A., and A. J. Wrobel (2002): "Boiteux's solution to the shifting-peak problem and the equilibrium price density in continuous time", Economic Theory, 20, 503-537.

[14] Horsley, A., and A. J. Wrobel (2002): "Efficiency rents of pumped-storage plants and their uses for operation and investment decisions", Journal of Economic Dynamics and Control, $27,109-142$. 
[15] Horsley, A., and A. J. Wrobel (2005): "Continuity of the equilibrium price density and its uses in peak-load pricing", Economic Theory, 26, 839-866.

http://www.springerlink.com/index/10.1007/s00199-004-0568-3

[16] Horsley, A., and A. J. Wrobel (2005): "The short-run approach to long-run equilibrium: a general theory with applications", CDAM Research Report LSE-CDAM-2005-02.

http://www.cdam.lse.ac.uk/Reports/reports2005.html

[17] Horsley, A., and A. J. Wrobel (2005): "Efficiency rents of a hydroelectric storage plant with a variable head", forthcoming CDAM Research Report, LSE.

[18] Ioffe, A. D., and V. M. Tihomirov (1979): Theory of extremal problems. Amsterdam-New York-Oxford: North-Holland.

[19] Jacoby, H. P. (1967): "Analysis of investments in electric power", Economic Development Series, Center for International Affairs, Harvard University.

[20] Koopmans, T. C. (1957): "Water storage policy in a simplified hydroelectric system", in: Proceedings of the First International Conference on Operational Research, pp. 193-227. London-Baltimore, 1957. (Cowles Foundation Paper No. 115. Also in: The collected papers of T. C. Koopmans, pp. 282-316.)

[21] Koopmans, T. C. (1977): "Concepts of optimality and their uses", American Economic Review, 67, 261-274 (Nobel Lecture 1975).

[22] Munasinghe, M., and J. J. Warford (1982): Electricity pricing: theory and case studies. Baltimore-London: The Johns Hopkins University Press (for the World Bank).

[23] Phu, H. X. (1987): "On the optimal control of a hydroelectric power plant", Systems and Control Letters, 8, 281-288.

[24] Rockafellar, R. T. (1974): Conjugate duality and optimization. Philadelphia, PA: SIAM.

[25] Tjur, T. (1972): On the mathematical foundations of probability (Lecture Notes 1). Institute of Mathematical Statistics, University of Copenhagen. 

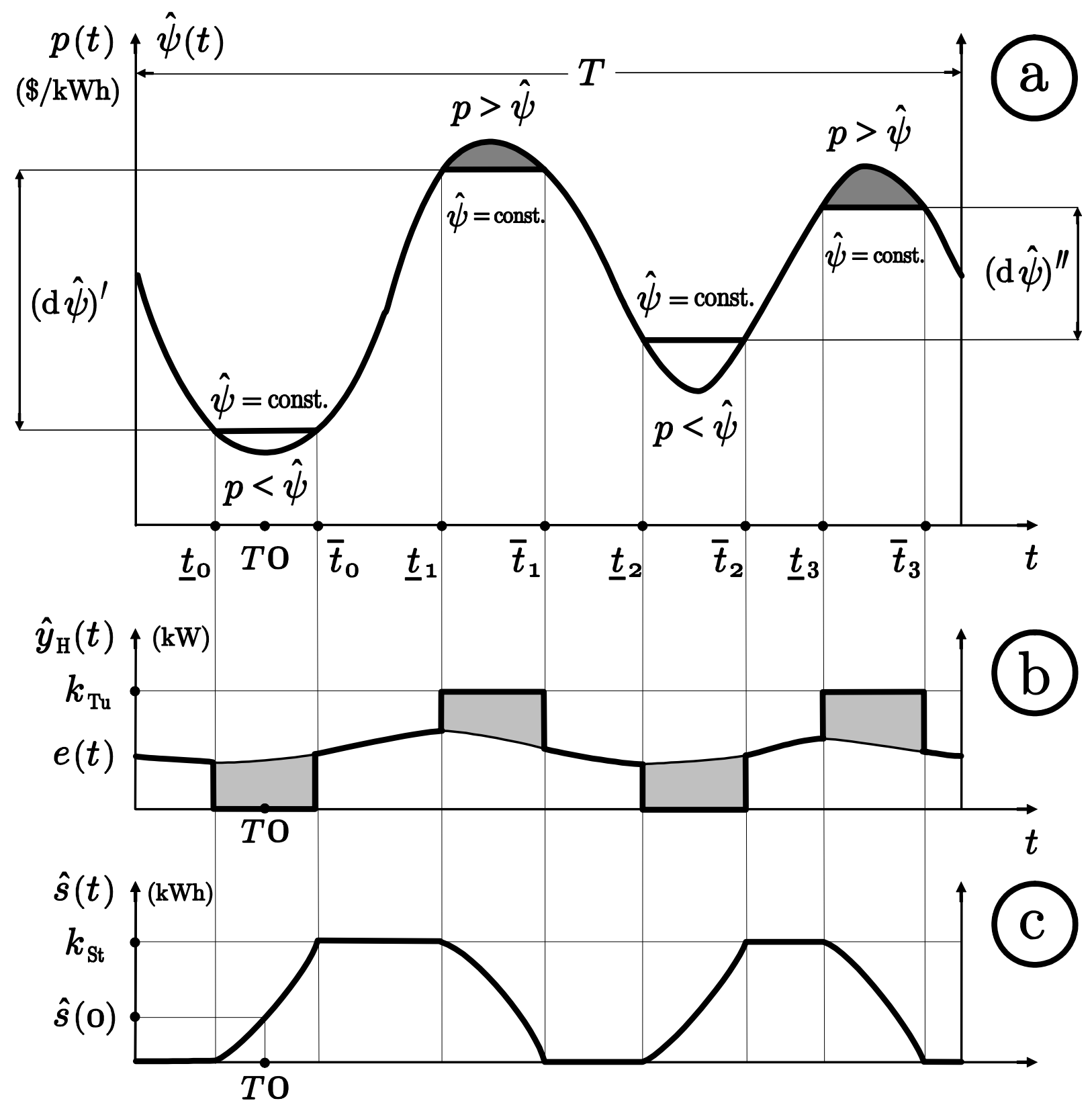

Figure 1: Trajectories of: (a) shadow price for water $\hat{\psi}$, (b) profit-maximising hydro output $\hat{y}_{\mathrm{H}}$, (c) water stock. Unit rent for storage capacity is $\operatorname{Var}_{\mathrm{c}}^{+}(\hat{\psi})=(\mathrm{d} \hat{\psi})^{\prime}+(\mathrm{d} \hat{\psi})^{\prime \prime}$, the sum of rises of $\hat{\psi}$. Unit rent for turbine capacity is $\int_{0}^{T}(p(t)-\hat{\psi}(t))^{+} \mathrm{d} t$, the sum of dark grey areas in (a). In (b), each of the light grey areas equals the reservoir's capacity $k_{\mathrm{St}}$. When $\hat{y}_{\mathrm{H}}(t) \neq e(t)$ in $(\mathrm{b})$, the thin line is the inflow trajectory $e$, and the thick line is $\hat{y}_{\mathrm{H}}$. 


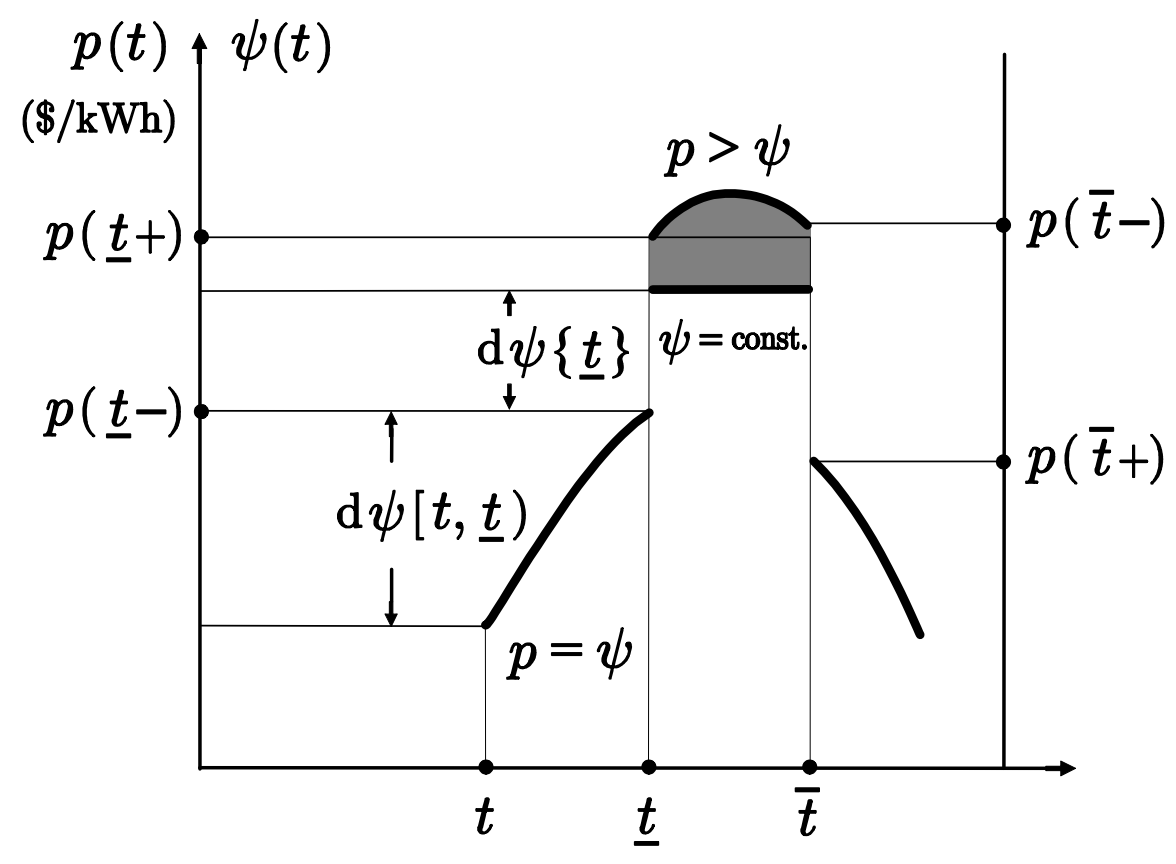

Figure 2: Indeterminacy of an optimal shadow price of water $\psi$ when the TOU price of good $p$ is discontinuous. In the case shown, the constant value of $\psi$ on $(\underline{t}, \bar{t})$ can be set at any level between $p(\underline{t}-)$ and $p(\underline{t}+)$; so the jump of $\psi$ at $\underline{t}$ is an indeterminate part of the reservoir's unit rent. The dark grey area represents $\int_{\underline{t}}^{\bar{t}}(p-\psi)^{+} \mathrm{d} t$, the interval's contribution to the turbine's unit rent. 